\title{
Relational Nullable Types with Boolean Unification
}

\author{
MAGNUS MADSEN and JACO VAN DE POL, Aarhus University, Denmark
}

\begin{abstract}
We present a simple, practical, and expressive relational nullable type system. A relational nullable type system captures whether an expression may evaluate to null based on its type, but also based on the type of other related expressions. The type system extends the Hindley-Milner type system with Boolean constraints, supports parametric polymorphism, and preserves principal types modulo Boolean equivalence. We show how to support full Hindley-Milner style type inference with an extension of Algorithm W.

We conduct a preliminary study of open source projects showing that there is a need for relational nullable type systems across a wide range of programming languages. The most important findings from the study are: (i) programmers use programming patterns where the nullability of one expression depends on the nullability of other related expressions, (ii) such invariants are commonly enforced with run-time exceptions, and (iii) reasoning about these programming patterns requires not only knowledge of when an expression may evaluate to null, but also when it may evaluate to a non-null value. We incorporate these observations in the design of the proposed relational nullable type system.
\end{abstract}

CCS Concepts: • Theory of computation $\rightarrow$ Program semantics.

Additional Key Words and Phrases: relational nullable type system, relational pattern matching, choose construct, type inference, Algorithm W, Boolean unification, successive variable elimination algorithm

ACM Reference Format:

Magnus Madsen and Jaco van de Pol. 2021. Relational Nullable Types with Boolean Unification. Proc. ACM Program. Lang. 5, OOPSLA, Article 110 (October 2021), 28 pages. https://doi.org/10.1145/3485487

\section{INTRODUCTION}

Null - infamously dubbed the "Billion Dollar Mistake" by its inventor Sir Tony Hoare - is a special value that is an inhabitant of every type. The null value serves two distinct purposes: to represent the absence of a value or as a place holder for an uninitialized value. Null is dangerous because any attempt to use it as an ordinary value, e.g. by dereference or invocation, triggers the dreaded NullPointerException crashing the program or, even worse, leading to undefined behavior, depending on the semantics of the programming language.

A nullable type system prevents such crashes or undefined behavior by tracking, for every expression, whether it may evaluate to null [Banerjee et al. 2019; Fähndrich and Xia 2007; Nieto et al. 2020b; Summers and Müller 2011]. If an expression is nullable then the programmer must explicitly check for null before the result of the expression can be used. If, on the other hand, an expression is non-nullable then the type system guarantees that the expression cannot evaluate to null at run-time. Thus the programmer does not have to check for null.

Recently, nullable type systems have become popular in mainstream programming languages. New programming languages such as Kotlin, Swift, and TypeScript have adopted nullable type systems from the start, whereas established languages such as C\# and Scala are being retrofitted with nullable type systems [Fähndrich and Leino 2003; Nieto et al. 2020b].

Authors' address: Magnus Madsen, magnusm@cs.au.dk; Jaco van de Pol, jaco@cs.au.dk, Department of Computer Science, Aarhus University, Åbogade 34, Aarhus, 8210, Denmark.

This work is licensed under a Creative Commons Attribution 4.0 International License.

(c) 2021 Copyright held by the owner/author(s).

2475-1421/2021/10-ART110

https://doi.org/10.1145/3485487

Proc. ACM Program. Lang., Vol. 5, No. OOPSLA, Article 110. Publication date: October 2021. 
In this paper we propose a novel relational nullable type system that captures not only nullability of a single expression, but its nullability with respect to other related expressions. The type system can enforce invariants such as: "the expressions $e_{1}$ and $e_{2}$ cannot both be null", "the expressions $e_{1}$, $e_{2}$, and $e_{3}$ cannot all be non-null", or: "among the expressions $e_{1}, e_{2}$, and $e_{3}$ exactly one must be null". For a more concrete example, imagine a method that takes two arguments: file and encoding where either both arguments must be null or both arguments must be non-null.

We conduct a preliminary study of open source projects showing that there is a need for relational nullable type systems across a wide range of programming languages. The most important findings are that programmers use programming patterns where the nullability of one expression depends on the nullability of other related expressions. Such programming patterns occur in imperative, object-oriented, and functional languages, and without type system support, programmers currently rely on runtime checks to enforce these relational nullability invariants.

To the best of our knowledge, contemporary nullable type systems, in the research literature and in mainstream programming languages, only consider the nullability of a single expression. That is, while several existing systems take into account that the nullability of an expression may vary over time (e.g. during object construction), no existing system considers that the nullability of one expression may depend on the nullability of other related expressions.

We propose a simple, practical, and expressive relational nullable type system to ensure the safety of such programming patterns. The type system captures both may and must information about nullity and non-nullity, i.e. whether an expression may or must evaluate to null and whether it may or must evaluate to a non-null value. The type system enables a special form of pattern matching on a sequence of expressions and their nullity. Unlike in a traditional pattern match, the cases of such a match do not have to be exhaustive. Instead, the type system prevents matching on expressions whose values could lead to an unmatched case at runtime. This is really the simplest solution for programming with null: simply leave out cases that should not occur and the type system ensures that they cannot occur.

An important property of the type system is that it supports full type inference. The key insight is that the exhaustiveness condition can be translated into a Boolean formula which can be inferred by an extension of Algorithm W because Boolean formulas have most general unifiers [Boole 1847; Madsen and van de Pol 2020; Martin and Nipkow 1989].

While our work is presented in the context of null, it is equally applicable to relational uses of the Option data type and it gives precise types to combinators such as filter, map, and flatMap. The type system extends the Hindley-Milner type system, supports parametric polymorphism and preserves principal types modulo Boolean equivalence.

In summary, the contributions of this paper are:

- (Preliminary Study) We conduct a preliminary study on the use of relational nullability programming patterns in open source projects. We find that such patterns occur across a wide range of programming languages (cf. Section 2).

- (Type System) We present a minimal calculus with null and a special pattern matching construct named choose. We present a declarative type system for the calculus based on Hindley-Milner extended with Boolean constraints and we show how to perform principal type inference using an extension of Algorithm W (cf. Section 3 and 4).

- (Implementation) We provide two implementations of the calculus and type system: a proofof-concept that exactly mirrors the formal system and an extension of the Flix programming language with relational nullable types (cf. Section 5).

In Section 6, we compare our contributions to related work on nullable type systems. 


\section{PRELIMINARY STUDY}

We now present a preliminary study on the use of relational nullability programming patterns in real-world open source projects. The primary purpose of the study is twofold: to ascertain whether: (i) there is a use case for relational nullable type systems, and (ii) to understand what features such a system should support. That is, we want to understand if there is a problem, but not necessarily the scope of the problem. We begin with a discussion of our methodology, next we present several representative examples of the programming patterns we found, and finally we present a summary of our most important quantitative findings.

\subsection{Methodology}

We used the www.grep.app website to search for relational nullability programming patterns in open source projects hosted on GitHub. We searched for strings that are indicative of relational nullable programming patterns. For example, the strings "cannot both be null" and "cannot both be non-null" which are likely to occur in comments or error messages. We also searched for many spelling variants of such strings, including "can't both be null' and "canot both be null" (sic). We also substitute "null' for "NULL", "nil", "None", "Some", and so forth. In addition to these natural language queries, we also searched for specific program fragments. For example, (None, None) $\Rightarrow$ (a fragment of a pattern match on two None values). We collected the results from the queries and manually inspected the results. We included a program fragment based on these selection criteria:

- the program fragment was a relational nullability programming pattern.

- the program fragment was readable (i.e. we excluded auto-generated or bizarre code.)

- the program fragment had an invariant within the reach of static type systems. ${ }^{1}$

- the program fragment was a not a duplicate (i.e. due to duplicated code on GitHub.)

- we limited the study to one program fragment per GitHub repository.

- we limited the study to programming languages with static type systems.

In total we collected 108 manually inspected program fragments that satisfied the above criteria. The program fragments are available in the supplementary material. The preliminary study is only the tip of the iceberg: The www.grep.app website only covers an estimated $0,5 \%$ of all public GitHub repositories and our simple textual queries are unlikely to discover all relevant program fragments. Yet, the study is still sufficient to demonstrate that there is a use case for relational nullable type systems and to help inform the design of such type systems.

\subsection{Selected Examples}

We now discuss a few representative examples of the collected program fragments. Next, we present the quantitative results of the study.

pmd/pmd (Java) pmd-core/src/main/java/net/sourceforge/pmd/util/database/DBType.java

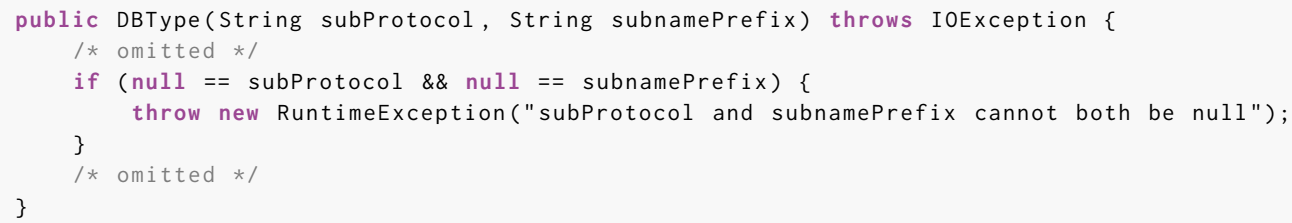

${ }^{1}$ For example, a program fragment that performs an RPC call over the network and expects a record with two null or non-null fields is out of scope. This assumption is in line with most work on nullable type systems. 
The above code fragment, from the pmd/pmd project, shows part of the DBType constructor. The constructor takes two arguments: subProtocol and subnamePrefix. If both are null the constructor throws a RuntimeException to abort execution.

\section{CloudburstMC/Nukkit (Java) src/main/java/cn/nukkit/OfflinePlayer.java}

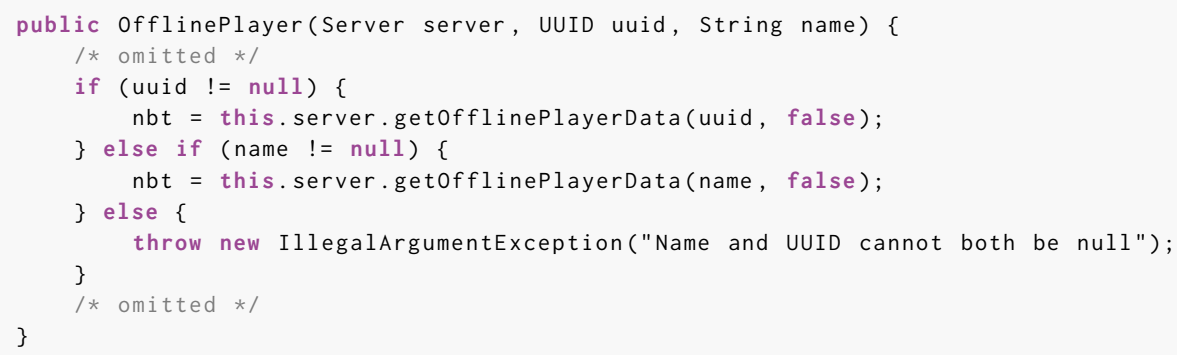

The above code fragment, from the CloudburstMC/Nukkit project, shows part of the OfflinePlayer constructor. The constructor takes three arguments: server, uuid, and name. If both uuid and name are null the constructor throws an IllegalArgumentException to abort execution.

apache/druid (Java) sql/src/main/java/org/apache/druid/sql/calcite/rel/Projection. java

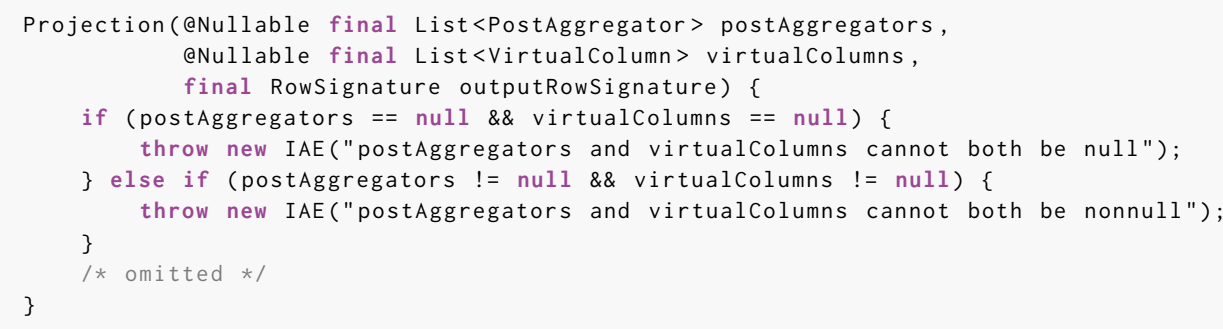

The above code fragment, from the apache/druid project, shows the Projection constructor which takes three arguments: postAggregators, virtualColumns, and outputRowSignature. If both postAggregators and virtualColumns are null then the constructor throws an IAE exception. Similarly, if both postAggregators and virtualColumns are non-null then the constructor throws an IAE exception. In other words, exactly one of the two arguments must be null.

openssl/openssl (C) crypto/ffc/ffc_params_generate.c

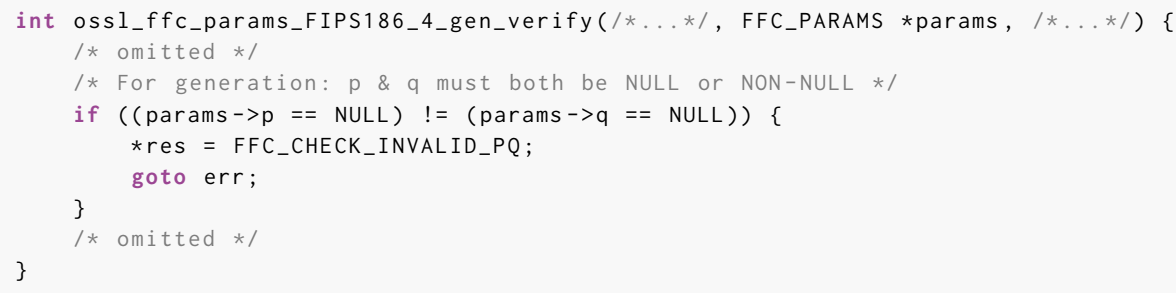

The above code fragment, from the openssl/openssl project, shows part of the ossl_ffc_params_FIPS186_4_gen_verify procedure. The procedure takes several parameters of which the params argument must be a struct with two fields: $p$ and $q$. The above fragment asserts that either: (i) both $p$ and $q$ are NULL or (ii) both $p$ and $q$ are non-NULL. 
torvalds/linux (C) include/drm/drm_atomic_helper.h

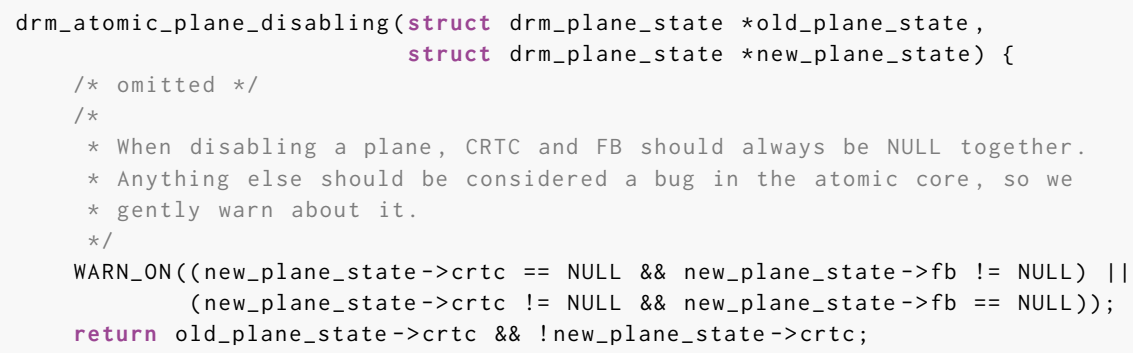

The above code fragment, from the torvalds/linux project, shows part of the drm_atomic_plane_disabling procedure. The procedure has a local variable new_plane_state which is a struct with two fields: crtc and fb. As the comment explains, if either field is NULL the other field must also be NULL. It is a bug if one field is NULL and the other is non-NULL.

rust-analyzer/rust-analyzer (Rust) crates/ide/src/completion/complete_record.rs

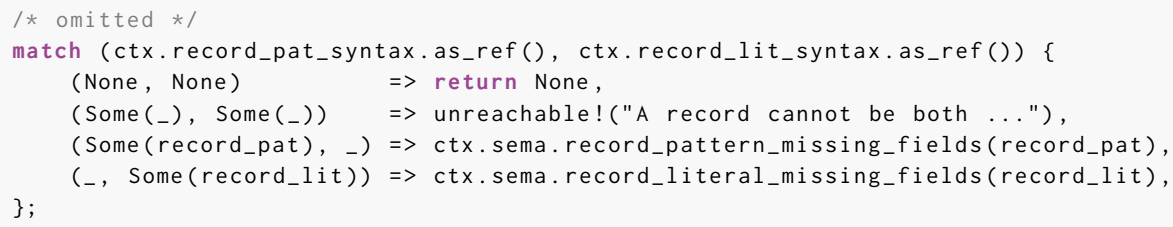

The above code fragment, from the rust-analyzer/rust-analyzer project, shows a program fragment that does a pattern match on the two fields: ctx.record_pat_syntax and ctx.record_lit_syntax. If both fields are None the function returns None. If one or the other is Some then the pattern match generates an error message. If both values are Some then the function triggers a "panic" using the unreachable! macro which aborts execution. As the Rust documentation explains ${ }^{2}$, a panic is serious and should only be used when a program reaches an unrecoverable state.

snowplow/iglu (Scala) 0 -common/igluctl/src/main/scala/.../ctl/commands/S3cp.scala

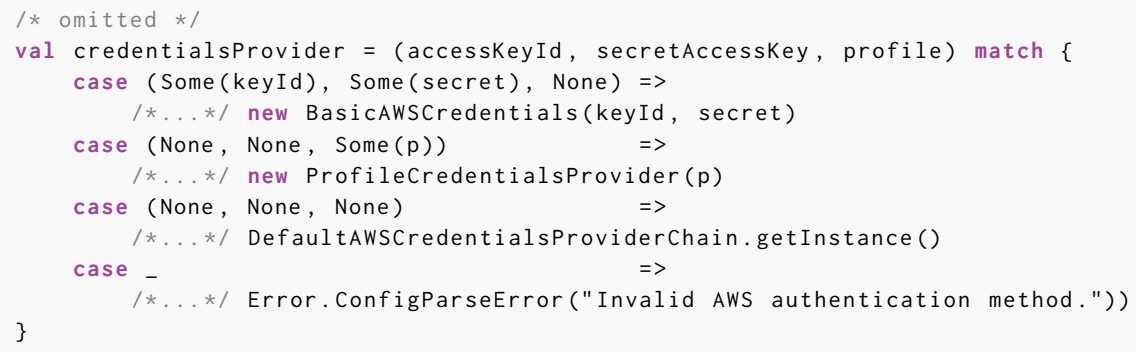

The above code fragment, from the snowplow/iglu project, shows a pattern match on three values: accessKeyld, secretAccessKey, and profile. The pattern match returns an error value if either: (i) accessKeyld is Some and secretAccessKey is None (or vise versa), or if (ii) accessKeyld, secretAccessKey, and profile are all Some.

\footnotetext{
${ }^{2}$ https://doc.rust-lang.org/std/macro.panic.html
} 


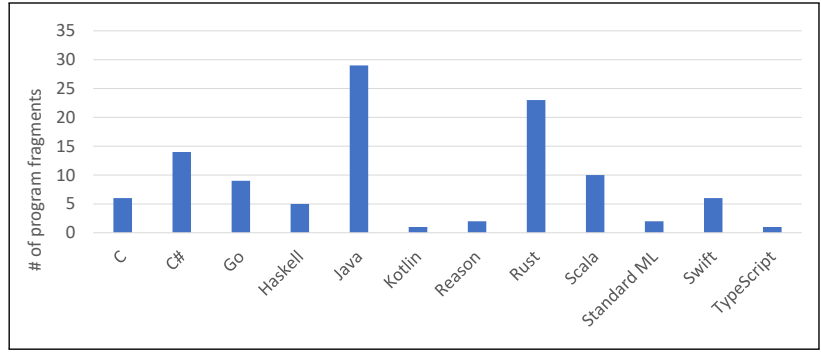

Fig. 1. Programming languages.

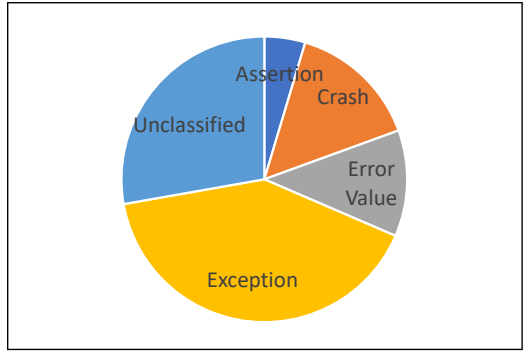

Fig. 2. Enforcement mechanism.

combust/mleap (Scala) mleap-core/src/main/scala/ml/combust/.../feature/StandardScalerModel.scala

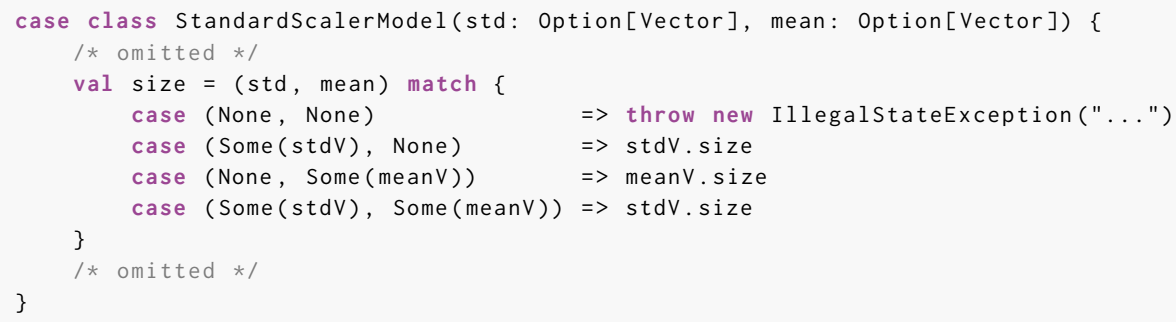

The above code fragment, from the combust/mleap project, shows the (inline) constructor for the StandardScalerModel class. The constructor takes two arguments: std and mean. If both are None then the constructor throws an IllegalStateException to abort execution.

\subsection{Preliminary Results}

We now present the quantitative results of the study. In total we found 108 program fragments that satisfied the selection criteria. The fragments are available in the supplementary material.

2.3.1 Programming Languages. Figure 1 shows a breakdown of the programming languages that each program fragment is written in. The figure shows that relational nullability programming patterns arise across a wide range of programming languages, including in languages that have null (e.g. C, C\#, Go, Java), languages that have the Option / Maybe data type (e.g. Haskell, Rust), and languages that have both null and Option (e.g. Scala). This is not too surprising: Inspection of the program fragments reveals that relational nullability is a property of the problem domain.

2.3.2 Enforcement Mechanism. Figure 2 shows a breakdown of the enforcement mechanisms used in the program fragments. Given that these programming languages do not have relational nullable type systems, some other mechanism must be used to enforce such invariants. We grouped these enforcement mechanisms into five categories: Assertion, Crash, Error Value, Exception, and Unclassified. Most of the categories are self-explanatory. The Crash category covers enforcement mechanisms more severe than exceptions, such as panic! in Rust and fatalError in Swift. The Error Value covers enforcement mechanisms where the programmer returned a "monadic" error value such as Err of the Result data type. We used the Unclassified category for everything else or when we were in doubt. The figure shows that most common enforcement mechanism is to throw an exception. Together, Assertion (4\%), Crash (15\%), and Exception (41\%) account for $60 \%$ of the enforcement mechanisms. Thus, in lieu of type system support, violations of relation nullability invariants cause run-time errors. 


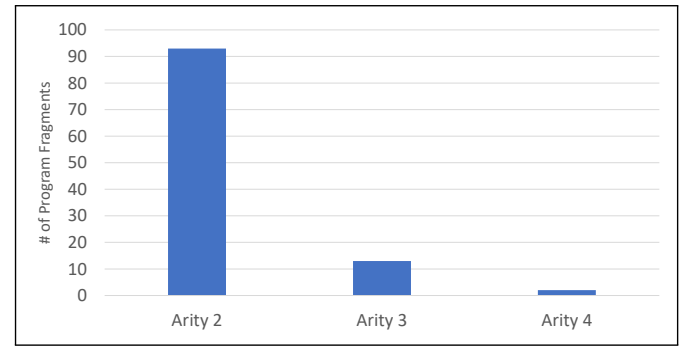

Fig. 3. Number of related expressions.

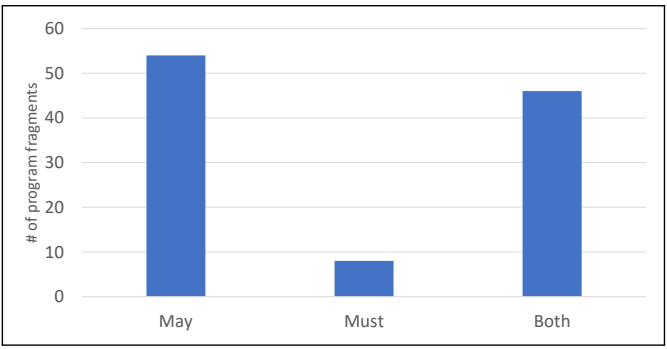

Fig. 4. Use of may and must information.

2.3.3 Related Expressions. Figure 3 shows a breakdown of the number of expressions whose nullability is inter-dependent. The figure shows that it is common to have two expressions whose nullity depend on each other. However, there are also cases where the nullity of three expressions depend on each other. We can imagine that as the number of related expressions grow the invariants may become too complex for the programmer to understand and use. However, with better type system support we believe that programmers might start to encode more complex domain invariants.

This a common theme in programming language design. The introduction of a new feature that makes it simpler and safer to use certain programming patterns may encourage the use of such patterns and lead to a new way of writing programs.

2.3.4 May and Must Nullity. Figure 4 shows a breakdown of how many of the program fragments require may and must information about the nullability or non-nullability of related expressions. A standard nullable type system can guarantee that an expression cannot evaluate to null, but it cannot enforce that an expression must evaluate to null. Thus it cannot capture a situation where if $e_{1}$ evaluates to null then $e_{2}$ must also evaluate to null. The figure shows that may knowledge is much more common than must knowledge. However, for approximately half of the program fragments, both may and must knowledge is required to capture the relational nullability invariants. This strongly suggests that a relational nullability type system must track two pieces of information: the conditions under which an expression evaluates to null and the conditions under which it evaluates to a non-null value.

\subsection{Summary of Findings}

We conclude with a summary of our most important observations:

- (Obs I): Programmers use programming patterns where the nullability of one expression depends on the nullability of other related expressions.

- (Obs II): Programmers use such programming patterns across a wide range of languages, including imperative, object-oriented, and functional languages.

- (Obs III): Reasoning about such patterns requires both may and must information.

- (Obs IV): Relational nullability typically depends on two or three expressions.

- (Obs V): In lieu of type system support, programmers currently rely on run-time checks to enforce relational nullability invariants.

Interestingly, in the initial design of our relational nullable type system, we only considered when an expression may evaluate to null, but the preliminary study demonstrated that it is equally important to also capture when an expression must evaluate to null. Without the study, we would have ended up with a less useful design. 
The above program is well-typed and prints " $\mathrm{x}$ and $\mathrm{y}$ are both null!" followed by " $\mathrm{x}$ and $y$ are both non-null!" when executed. The inferred type of the function $f$ ensures that it is a type error to call it with a null and a non-null value (or vise versa).

Example $V$. If we modify the above program to:

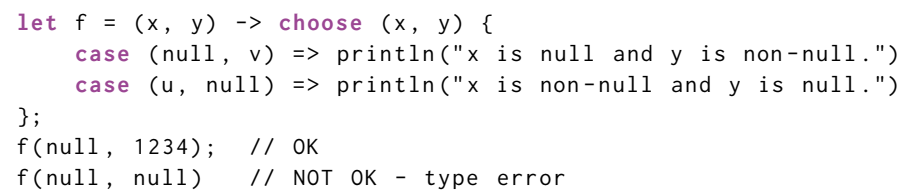

then the inferred type of the function $f$ ensures that it can only be invoked with two values: exactly one of which must be null and the other must be non-null.

As these examples demonstrate, an important property of our system is that:

Given a pattern match on the expressions $e_{1}, \cdots, e_{n}$, the choose construct permits matching on any subset of cases. If a case is omitted the type system enforces that the types of $e_{1}, \cdots, e_{n}$ cannot give rise to that case at run-time.

A corollary is that an exhaustive pattern match does not impose any requirements on the nullity of $e_{1}, \cdots, e_{n}$. Conversely, an empty pattern match enforces that $e_{1}, \cdots, e_{n}$ must be uninhabited.

\subsection{Type System}

We now informally describe how the type system works. The typing judgement of an expression is of the form $\Gamma \vdash e: \pi$ ? $(\varphi, \psi)$, where $\pi$ is a proper type (e.g. String), and $\varphi$ and $\psi$ are Boolean formulas, called the nullity formulas of the type. The Boolean formula $\varphi$ captures when the expression may evaluate to null, while $\psi$ captures when the expression may evaluate to a non-null value.

Consequently, the values null and non-null have the following types (for any $\varphi$ and $\psi$ ):

$$
\text { null : } \alpha ?(\mathrm{~T}, \psi) \quad v: \pi ?(\varphi, \mathrm{T})
$$

If we have an expression with a type of the form $\pi ?(\mathrm{~F}, \psi)$ (for any $\pi, \psi)$ then the expression cannot evaluate to null. Similarly, if we have an expression with a type of the form $\pi ?(\varphi, F)$ (for any $\pi, \varphi)$ then the expression cannot evaluate to a non-null value. If we have an expression with a type of the form $\pi$ ? (T, T) then we have no information; the expression could evaluate to null or to a non-null value. For example, the expression "if $c$ then null else 123" has the type Int ? (T, T) because the type system combines the information from the two branches. Finally, if an expression has a type of the form $\pi$ ? (F, F) then it cannot evaluate to any value at all; the type is uninhabited.

Let us now consider what the type of the function on the left should be:

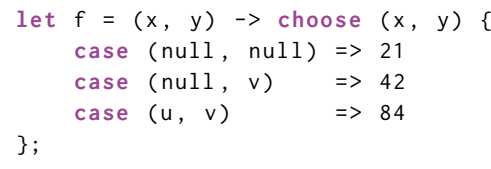

$$
P=\left(\begin{array}{ll}
0 & 0 \\
0 & 1 \\
1 & 1
\end{array}\right) \Rightarrow\left(\begin{array}{ll}
0 & \square \\
\square & 1
\end{array}\right)=\operatorname{Saturate}(P)
$$

For simplicity, let us assume that the types of $\mathrm{x}$ and $\mathrm{y}$ are of the form:

$$
x: \pi_{1} ?\left(X_{0}, X_{1}\right) \quad y: \pi_{2} ?\left(Y_{0}, Y_{1}\right)
$$

where $\pi_{1}$ and $\pi_{2}$ are proper types, and the Boolean variables $\left(X_{0}, X_{1}\right)$ and $\left(Y_{0}, Y_{1}\right)$ indicate the nullity formulas of $\mathrm{x}$ and $\mathrm{y}$.

We want to translate the pattern match into a constraint on $X_{0}, X_{1}, Y_{0}, Y_{1}$ that captures when the pattern match is defined. The matrix $P$, shown above, represents the value vectors matched 
by the pattern match, where 0 represents null, 1 represents a non-null value, and $\square$ represents a wildcard. Note that an expression like (null, if $c$ then null else 123) corresponds to $(0, \square)$, which is not covered by a single row of $P$, although its concrete instances $(0,0)$ and $(0,1)$ are covered. To remedy this, a saturation procedure (cf. Section 4.6), combines any two rows that differ in a single entry. The result matrix Saturate $(P)$ covers all value vectors where either $\mathrm{x}$ is definitely null (i.e. $\neg X_{1}$ ), or $\mathrm{y}$ is definitely non-null (i.e. $\neg Y_{0}$ ). Thus the exhaustiveness constraint is given by the side condition $\neg X_{1} \vee \neg Y_{0}$.

How do we handle the side condition? At this point, we call a Boolean unification procedure on the unification problem $\neg X_{1} \vee \neg Y_{0} \stackrel{?}{=} \mathrm{T}$. This yields a most general unifier, for instance $\left[Y_{0} \mapsto Y_{0} \wedge \neg X_{1}\right]$. We can now give the following type to the function $f$ :

$$
f: \pi_{1} ?\left(X_{0}, X_{1}\right) \rightarrow \pi_{2} ?\left(Y_{0} \wedge \neg X_{1}, Y_{1}\right) \rightarrow \text { Int } ?(\mathrm{~F}, \mathrm{~T})
$$

To understand why this is correct, let us consider what happens if we try to call $f$ where the first argument is non-null. In this case, we know that $X_{1}=\mathrm{T}$ and thus the type of the second argument simplifies to $\pi_{2}$ ? $\left(\mathrm{F}, Y_{1}\right)$. But now the constraint on the second argument ensures that it cannot be null! Indeed, the pattern match has no case for a (non-null, null) pair.

\section{CALCULUS}

We now present $\lambda_{\text {null }}^{\text {rel }}$ : a lambda calculus with a relational nullable type system. We then present an extension of Algorithm W that enables Hindley-Milner style type inference. We begin with a brief introduction to Boolean unification which is the key technique that enables type inference.

\subsection{Preliminaries: Boolean Unification}

Boolean Formulas. Let $\mathcal{B}=\{\mathrm{T}, \mathrm{F}\}$ denote the Boolean values true and false. Let BoolVar denote a set of Boolean variables $(\beta \cdots)$. The set of Boolean formulas is then defined inductively as:

$$
\varphi, \psi \in \text { Formula }=\beta|\mathrm{T}| \mathrm{F}|\neg \varphi| \varphi \wedge \varphi \mid \varphi \vee \varphi
$$

A valuation $V$ is a mapping from Boolean variables to $\{\mathrm{T}, \mathrm{F}\}$. Valuations are extended to evaluate formulas, by means of the standard truth tables. We write $\varphi \equiv_{\mathbb{B}} \psi$ if $V(\varphi)=V(\psi)$ for all valuations $V$ (i.e. $\varphi$ and $\psi$ are logically equivalent). For example, we have:

$$
\mathrm{T} \equiv_{\mathbb{B}} \neg \mathrm{F} \quad x \equiv_{\mathbb{B}} \neg(\neg x) \quad x \wedge y \equiv_{\mathbb{B}} y \wedge x \quad x \wedge \neg x \equiv_{\mathbb{B}} \mathrm{F} \wedge z
$$

Note that two formulas do not have to share the same variables to be equivalent. We write $\varphi \Rightarrow \psi$ if $\varphi$ logically implies $\psi$. That is, for all valuations $V$, if $V(\varphi)=\mathrm{T}$ then $V(\psi)=\mathrm{T}$.

Substitutions. A Boolean substitution $S$ is a partial mapping BoolVar $\hookrightarrow$ Formula. We write $\left\{\beta_{i} \mapsto \varphi_{i}\right\}_{i \in I}$ for the substitution $S$, such that $S\left(\beta_{i}\right)=\varphi_{i}$. We extend substitutions to total functions on formulas, and write $\varphi S$ for the result of applying $S$ to $\varphi$. The composition $S_{1} S_{2}$ of two substitutions $S_{1}$ and $S_{2}$ is defined such that $\varphi\left(S_{1} S_{2}\right)=\left(\varphi S_{1}\right) S_{2}$. We write $S_{1} \leq_{\mathbb{B}} S_{2}$ (substitution $S_{1}$ is more general than $S_{2}$ ) iff there exists a substitution $S_{3}$ such that for all $x \in \beta, x S_{1} S_{3} \equiv_{\mathbb{B}} x S_{2}$.

Definition 4.1 (Boolean Unification). Given formulas $\varphi$ and $\psi$ the Boolean Unification Problem $\varphi \stackrel{?}{=} \psi$ is to compute a solution, i.e., a substitution $S$ such that $\varphi S \equiv_{\mathbb{B}} \psi S$, or to report that no solution exists. A solution $S$ is a most general unifier (mgu), if $S \leq_{\mathbb{B}} S^{\prime}$ for all other solutions $S^{\prime}$.

Note that the most general unifier is not unique, not even modulo $\equiv_{\mathbb{B}}$, but importantly the set of all solutions can be represented by a single unifier, i.e. the Boolean unification problem is unitary [Martin and Nipkow 1989].

Theorem 4.2 (MARTin AND Nipkow [1989]). Boolean Unification is decidable. Moreover, if $\varphi \stackrel{?}{=} \psi$ is solvable then there exists a most general unifier. 
The original result goes back Boole himself [Boole 1847]. The most general unifier can be computed by the Successive Variable Elimination (SVE) algorithm [Martin and Nipkow 1989] or Löwenheim's algorithm [Löwenheim 1908]. In this paper, we shall not concern us with the specific implementation details of these algorithms, but instead we refer the reader to [Martin and Nipkow 1989] and [Madsen and van de Pol 2020]. The existence of most general unifiers and algorithms to compute them is what enables us to extend Algorithm W and ultimately to build a type inference algorithm for the proposed relational nullable type system.

We now present a few examples of Boolean unification problems. We begin with:

$$
\begin{array}{lll}
x \wedge y \stackrel{?}{=} \mathrm{T} & \text { has mgu } & S=\{x \mapsto \mathrm{T}, y \mapsto \mathrm{T}\} \\
x \wedge y \stackrel{?}{=} \mathrm{F} & \text { has mgu } & S=\{x \mapsto x \wedge \neg y\}
\end{array}
$$

The substitution $\{x \rightarrow \mathrm{F}, y \rightarrow \mathrm{T}\}$ is another unifier for Example II, but it is not most general. Other unifiers are $\{x \rightarrow \mathrm{T}, y \rightarrow \mathrm{F}\}$ and $\{x \rightarrow \neg y\}$, but none of these is most general, and all are instances of the most general unifier given above. Note that the unifier is equi-general to another most-general unifier $\{y \rightarrow y \wedge \neg x\}$. There can be many unifiers and several most general unifiers, but once we have one most general unifier, we can obtain all other unifiers by instantiating it.

The seemingly more complicated unification problem:

$$
x \vee y \stackrel{?}{=} x \wedge y \quad \text { has mgu } S=\{x \mapsto y\}
$$

(Example III)

However, not all Boolean unification problems have solutions:

$$
\begin{aligned}
\mathrm{T} \stackrel{?}{=} \mathrm{F} & \text { cannot be unified } . \\
x \stackrel{?}{=} \neg x & \text { cannot be unified } .
\end{aligned}
$$

(Example V)

\subsection{Syntax and Semantics}

Syntax. The syntax of the $\lambda_{\text {null }}^{\text {rel }}$ calculus (cf. Figure 5a) includes the standard lambda calculus constructs: variables, constants, lambda abstractions, and function applications. The let-expression allows polymorphic generalization, as is standard in Hindley-Milner. We include the if-then-else expression to illustrate how the type system merges information from control-flow paths. Pairs and projections illustrate that we can handle nested data types. The null value is our raison d'être The expression choose $\overline{e_{m}}\left\{P \Rightarrow \overline{e_{b}}\right\}$ enables pattern matching on a sequence of expressions. Note that we use $\bar{x}$ throughout the paper to denote finite sequences of the proper length.

$$
\begin{aligned}
v \in \text { Val }= & c|\lambda x \cdot e|(v, v) \mid \text { null } \\
e \in \operatorname{Exp}= & x|v| e e \\
\mid & \text { let } x=e \text { in } e \\
\mid & \text { if } e \text { then } e \text { else } e \\
& (e, e) \mid \text { fst } e \mid \text { snd } e \\
\mid & \text { choose } \overline{e_{m}}\left\{P \Rightarrow \overline{e_{b}}\right\} \\
P \in \text { Mat }= & m \times n \text { matrix of } P a t \\
p \in P a t= & \square|x| \text { null } \\
c \in C s t= & \text { a set of constants } \\
x, y \in \text { Var }= & \text { a set of variable symbols }
\end{aligned}
$$
(a) Syntax of $\lambda_{\text {null }}^{\text {rel }}$.
(b) Types and Type Schemes of $\lambda_{\text {null }}^{\text {rel }}$.

$$
\begin{aligned}
\varphi \in \text { Formula } & =\mathrm{T}|\mathrm{F}| \beta|\neg \varphi| \varphi \wedge \varphi \mid \varphi \vee \varphi \\
\pi \in \text { ProperType } & =\alpha|\iota| \tau \rightarrow \tau \mid \tau \times \tau \\
\tau \in \text { NullableType } & =\pi ?(\varphi, \psi) \\
\sigma \in \text { Scheme } & =\tau|\forall \alpha . \sigma| \forall \beta . \sigma \\
\iota \in \text { BaseType } & =\text { Unit } \mid \text { Bool } \mid \text { Nat } \mid \text { String } \mid \cdots \\
\alpha \in \text { TypeVar } & =\text { a set of proper type variables } \\
\beta \in \text { BoolVar } & =\text { a set of Boolean variables }
\end{aligned}
$$

Fig. 5. Syntax and Types of $\lambda_{\text {null }}^{\text {rel }}$ 


$\begin{array}{cr}(\lambda x . e) v \rightarrow e[x \mapsto v] & (\text { E-ApP }) \\ \text { let } x=v \text { in } e \rightarrow e[x \mapsto v] & \text { (E-LET) } \\ \text { if true then } e_{2} \text { else } e_{3} \rightarrow e_{2} & (\text { E-ITE-1) } \\ \text { if false then } e_{2} \text { else } e_{3} \rightarrow e_{3} & (\text { E-ITE-2) } \\ \text { fst }\left(v_{1}, v_{2}\right) \rightarrow v_{1} & \text { (E-Fst) } \\ \text { snd }\left(v_{1}, v_{2}\right) \rightarrow v_{2} & \text { (E-SND) }\end{array}$

$$
\begin{aligned}
& \frac{\text { smallest } i \text { s.t. } S \triangleq \text { matchRow }\left(P_{i}, \bar{v}\right)}{\text { choose } \bar{v}\left\{P \Rightarrow \overline{e_{b}}\right\} \rightarrow e_{b}^{i} S} \text { (E-CHOosE) } \\
& \operatorname{matchRow}(\bar{p}, \bar{v})=\bigcup_{i} \operatorname{matchPat}\left(p_{i}, v_{i}\right) \text { (M-Row) } \\
& \operatorname{matchPat}(\varpi, v)=[] \\
& \operatorname{matchPat}(\text { null, null })=[] \\
& v \neq \text { null } \\
& \overline{\operatorname{matchPat}(x, v)=[x \mapsto v]}
\end{aligned}
$$

Fig. 6. Evaluation Rules of $\lambda_{\text {null }}^{\text {rel }}$

The choose $\overline{e_{m}}\left\{P \Rightarrow \overline{e_{b}}\right\}$ expression is akin to a pattern match. The expression consists of the following components: The vector of match expressions $\overline{e_{m}}$, the pattern matrix $P$, and the vector of expression bodies $\overline{e_{b}}$. We shall write $P_{i}$ for the $i^{\text {th }}$ row of the pattern matrix. We require that the length of the match expression vector $\overline{e_{m}}$ matches (no pun intended) the length of each row in the pattern matrix $P$, i.e. $\left|\overline{e_{m}}\right|=\left|P_{i}\right|$. We also require that the number of rows equals the length of the body vector, i.e. $|P|=\left|\overline{e_{b}}\right|$. The entries of the pattern matrix consist of three types of patterns: A wildcard $\square$ pattern, a null pattern, and a non-null pattern: variable $x$. This opens a local scope for $x$, which can only be used in the corresponding body $e_{b}^{i}$. We require that patterns are linear, i.e. a variable cannot occur twice in the same pattern row $P_{i}$.

A choose expression differs from a normal pattern match in three important ways: (i) a variable pattern exclusively matches non-null values, (ii) patterns cannot be nested, and (iii) patterns do not have to be exhaustive. Note that a consequence of (i) is that a wildcard pattern is not equivalent to a variable pattern with a fresh variable name.

Semantics. The semantics of $\lambda_{\text {null }}^{\text {rel }}$ is based on a call-by-value small-step operational semantics for the lambda calculus. Figure 6 shows the evaluation rules of $\lambda_{\text {null }}^{\text {rel }}$. The evaluation rules are standard. We write $e[x \mapsto v]$ for the capture avoiding substitution of $x \mapsto v$ into $e$, and extend it to simultaneous substitutions $e\left\{x_{1} \mapsto v_{1}, \ldots, x_{n} \mapsto v_{n}\right\}$. We omit the congruence rules, but intuitively evaluation always proceeds from left to right. The only rule that warrants discussion is the evaluation rule for the choose construct.

The (E-CHoosE) rule states that if we have a choose expression where the match expression vector has been reduced to a value vector $\bar{v}$ then we look for the first row $P_{i}$ in the pattern matrix where $P_{i}$ matches $\bar{v}$. The partial function matchRow $(\bar{p}, \bar{v})$ is defined when the value vector $\bar{v}$ matches the pattern vector $\bar{p}$. Otherwise, if there is no match between $\bar{v}$ and $\bar{p}$, the function is undefined. When defined, the function returns a substitution of the variables in the pattern vector. If row $i$ is the first row where a pattern match is found, then the choose expression reduces to the match body expression $e_{b}^{i}$ with the substitution $S$ applied to it. If there is no match, the choose expression is stuck. We shall show that the type system ensures that no expression ever gets stuck.

The (M-Row) rule defines the partial function matchRow $(\bar{p}, \bar{v})$ as the union of the substitutions of matchPat $\left(p_{i}, v_{i}\right)$ for each pattern and value pair. We know from our syntactic requirement that the length of $\bar{p}$ and $\bar{v}$ are the same. Moreover, we can safely compute the union of the substitutions returned by matchPat $\left(p_{i}, v_{i}\right)$ because the variables in $\bar{p}$ are assumed to be disjoint (i.e. all patterns are linear). If the partial function matchPat is undefined for any $\left(p_{i}, v_{i}\right)$ then matchRow is undefined for the whole vector $(\bar{p}, \bar{v})$. The rules (M-WILD), (M-NuLL), and (M-VAR) define when a pattern 
$p$ matches a value $v$. The rules are straightforward: A wildcard matches anything (but does not bind any variable), the null pattern matches only the null value (and does not bind any variable), and finally a variable pattern $x$ matches any non-null value and binds $x$ to that value. In other words, the matchPat function is undefined in two cases: (i) when the pattern is null and the value is non-null, and (ii) when the pattern is a variable $x$ and the value is null.

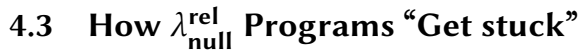

We briefly illustrate how $\lambda_{\text {null }}^{\text {rel }}$ programs may get stuck during evaluation. The obvious reason is when null is applied as a function, or when null is used as a condition. Another reason why a program gets stuck is if in a choose expression none of the cases apply to the match expressions:

$$
\begin{gathered}
\text { choose null }\{\operatorname{case} x \Rightarrow e\} \\
\text { choose }(123, \text { null })\{\operatorname{case}(\varpi, y) \Rightarrow e\} \\
\text { choose (null, null) }\left\{\operatorname{case}(\varpi, y) \Rightarrow e_{1}, \operatorname{case}(x, \varpi) \Rightarrow e_{2}\right\}
\end{gathered}
$$

In Example I, the variable pattern does not match the null value. In Example II, the value vector (123, null) does not match the single case in the pattern match. In Example III, the value vector (null, null) does not match any of the two cases in the pattern match.

\subsection{Declarative Type System}

Mono Types. The types of $\lambda_{\text {null }}^{\text {rel }}$ are separated into proper types $\pi$ and nullable types $\tau$, cf. Fig. $5 \mathrm{~b}$. The proper types and nullable types are defined by mutual induction. The proper types $\pi$ include types variables $\alpha$, a set of base types $\iota$ (e.g. Bool, Int, etc.), function types $\tau_{1} \rightarrow \tau_{2}$, and product types $\tau_{1} \times \tau_{2}$. Nullable types $\tau$ are triples of the form $\pi ?(\varphi, \psi)$ where $\pi$ is a proper type, and $\varphi$ and $\psi$ are two Boolean formulas that capture the nullity of the type. Intuitively, $\varphi$ and $\psi$ capture when an expression may evaluate to null and may evaluate to a non-null value. If $\varphi \equiv_{\mathbb{B}} \mathrm{F}$, the expression cannot evaluate to null. Similarly, if $\psi \equiv_{\mathbb{B}} \mathrm{F}$, the expression cannot evaluate to a non-null value.

We extend Boolean equivalence to proper and nullable types, as the smallest relation such that:

- If $\tau_{1} \equiv_{\mathbb{B}} \tau_{1}^{\prime}$ and $\tau_{2} \equiv_{\mathbb{B}} \tau_{2}^{\prime}$ then both $\left(\tau_{1}, \tau_{2}\right) \equiv_{\mathbb{B}}\left(\tau_{1}^{\prime}, \tau_{2}^{\prime}\right)$ and $\tau_{1} \rightarrow \tau_{2} \equiv_{\mathbb{B}} \tau_{1}^{\prime} \rightarrow \tau_{2}^{\prime}$

- If $\pi \equiv_{\mathbb{B}} \pi^{\prime}, \varphi \equiv_{\mathbb{B}} \varphi^{\prime}$, and $\psi \equiv_{\mathbb{B}} \psi^{\prime}$ then $\pi ?(\varphi, \psi) \equiv_{\mathbb{B}} \pi^{\prime} ?\left(\varphi^{\prime}, \psi^{\prime}\right)$

A substitution $S$ is a mapping in (TypeVar $\rightarrow$ ProperType $) \cup($ BoolVar $\rightarrow$ Formula), mapping type variables to proper types and Boolean variables to formulas. We define $\tau S=\tau^{\prime}$, the application of substitution $S$ to type $\tau$, in a standard way. In such a case, $\tau$ is said to be more general than $\tau^{\prime}$. We write $S_{0} S_{1}$ for the substitution such that $\tau\left(S_{0} S_{1}\right)=\left(\tau S_{0}\right) S_{1}$.

Type Schemes. Type schemes $\sigma$ extend nullable types by quantification over proper type variables $\alpha$ and Boolean variables $\beta$. That is, a type scheme is of the form $\forall \bar{\gamma}$. $\tau$, where $\bar{\gamma}$ is a vector of proper type variables and Boolean variables. Figure $5 \mathrm{~b}$ shows the types and type schemes of $\lambda_{\text {null }}^{\text {rel }}$.

We define $\mathrm{ftv}(\sigma)$ as the variables that occur free in $\sigma$. These include unbound proper type variables $\alpha$ and unbound Boolean variables $\beta$. We extend substitutions to capture-avoiding substitutions on type schemes (written $\sigma S$ ). We write $\sigma \sqsubseteq \tau$ (type $\tau$ is an instance of type scheme $\sigma$ ) if $\sigma=\forall \bar{\gamma} \tau^{\prime}$ and $\tau^{\prime} S=\tau$ for some substitution $S$ with $\operatorname{dom}(S)=\bar{\gamma}$.

Type Rules. Figure 7 shows the declarative type rules of $\lambda_{\text {null }}^{\text {rel }}$. A declarative typing judgement is of the form $\Gamma r_{d} e: \tau$. As is standard, the context $\Gamma:$ Var $\hookrightarrow$ Scheme is a partial function from variables to type schemes. We extend substitutions to contexts $(\Gamma S)$ and define $\mathrm{ftv}(\Gamma)$ as the union of all free type variables in the range of $\Gamma$. The type judgement $\Gamma \vdash_{d} e: \tau$, which expands to $\Gamma \vdash_{d} e: \pi ?(\varphi, \psi)$, states that the expression $e$ has proper type $\pi$ under type environment $\Gamma$ with nullity formulas $\varphi$ and $\psi$. Most of the type rules are straightforward. 


$$
\begin{aligned}
& \Gamma \vdash_{d} e: \tau \\
& \frac{\Gamma \vdash_{d} e: \tau_{1} \quad \tau_{1} \equiv \mathbb{B} \tau_{2}}{\Gamma \vdash_{d} e: \tau_{2}} \\
& \frac{(x, \sigma) \in \Gamma \quad \sigma \sqsubseteq \tau}{\Gamma \vdash_{d} x: \tau} \\
& \frac{\operatorname{typeOf}(c)=\iota}{\Gamma \vdash_{d} c: \iota ?(\varphi, \mathrm{T})} \\
& \overline{\Gamma r_{d} \text { null }: \pi ?(\mathrm{~T}, \psi)} \\
& \frac{\Gamma \vdash_{d} e: \tau_{1} \times \tau_{2} ?(\mathrm{~F}, \psi)}{\Gamma \vdash_{d} \text { fst } e: \tau_{1}} \\
& \frac{\Gamma \vdash_{d} e: \tau_{1} \times \tau_{2} ?(\mathrm{~F}, \psi)}{\Gamma \vdash_{d} \operatorname{snd} e: \tau_{2}} \\
& \frac{\Gamma \vdash_{d} e_{1}: \tau_{1} \quad \Gamma \vdash_{d} e_{2}: \tau_{2}}{\Gamma \vdash_{d}\left(e_{1}, e_{2}\right):\left(\tau_{1} \times \tau_{2}\right) ?(\varphi, \mathrm{T})} \\
& \frac{\Gamma, x: \tau_{1} \vdash_{d} e: \tau_{2}}{\Gamma \vdash_{d} \lambda x . e:\left(\tau_{1} \rightarrow \tau_{2}\right) ?(\varphi, \mathrm{T})} \\
& \frac{\Gamma \vdash_{d} e_{1}:\left(\tau_{1} \rightarrow \tau_{2}\right) ?(\mathrm{~F}, \psi) \quad \Gamma \vdash_{d} e_{2}: \tau_{1}}{\Gamma \vdash_{d} e_{1} e_{2}: \tau_{2}}(\mathrm{~T}-\mathrm{APP}) \\
& \frac{\Gamma \vdash_{d} e_{1}: \tau_{1} \quad \Gamma, x: \operatorname{gen}\left(\Gamma, \tau_{1}\right) \vdash_{d} e_{2}: \tau_{2}}{\Gamma \vdash_{d} \text { let } x=e_{1} \text { in } e_{2}: \tau_{2}}(\text { T-LET }) \\
& \text { (T-NuLL) } \\
& \frac{\Gamma \vdash_{d} e_{1}: \text { Bool } ?(\mathrm{~F}, \psi) \quad \Gamma \vdash_{d} e_{2}: \tau \quad \Gamma \vdash_{d} e_{3}: \tau}{\Gamma \vdash_{d} \text { if } e_{1} \text { then } e_{2} \text { else } e_{3}: \tau} \\
& \Gamma \vdash_{d} e_{m}^{j}: \pi_{j} ?\left(\varphi_{j}, \psi_{j}\right) \\
& \Gamma, \operatorname{ext}\left(\Gamma, P_{i}\right) \vdash_{d} e_{b}^{i}: \tau \\
& \frac{P \text { is exhaustive for }(\bar{\varphi}, \bar{\psi})}{\Gamma \vdash_{d} \text { choose } \overline{e_{m}}\left\{P \Rightarrow \overline{e_{b}}\right\}: \tau} \text { (T-Choose) } \\
& \operatorname{gen}(\Gamma, \tau)=\forall \bar{\gamma} \cdot \tau \text { where } \bar{\gamma}=\mathrm{ftv}(\tau) \backslash \mathrm{ftv}(\Gamma) \\
& \operatorname{ext}\left(\Gamma, P_{i}\right)=\left\{x_{j}: \pi_{j} ?\left(\chi_{i, j}, \mathrm{~T}\right) \mid P_{i, j}=x_{j}\right\}
\end{aligned}
$$

Fig. 7. Declarative Type Rules for $\lambda$ null. A typing judgement is of the form $\Gamma \vdash_{d} e: \tau$ where $\tau$ is nullable type. The expanded form is $\Gamma r_{d} e: \pi ?(\varphi, \psi)$ where $\pi$ is a proper type and $\varphi$ and $\psi$ are Boolean formulas.

The (T-EQ) rule states that if an expression $e$ can be typed as $\tau_{1}$, that type can be replaced by any equivalent type $\tau_{2} \equiv_{\mathbb{B}} \tau_{1}$. The (T-VAR) rule is the standard Hindley-Milner instantiation rule. It states that if the assumption $x: \sigma$ is in the context, then we can instantiate $\sigma$ to a specific type $\tau$, and conclude $x: \tau$. The (T-CsT) rule states that constants can be assigned a base type $\iota$, according to the typeOf function. The nullity $(\varphi, \mathrm{T})$ encodes that a constant is not null: the constant may evaluate to non-null. The polymorphic $\varphi$ indicates it may or may not evaluate to null. This is always sound, and the flexibility in choosing $\varphi$ allows the constant to occur in a branch together with null-values. The (T-NULL) rule states that the polymorphic type of null is any proper type $\pi$ with nullity formulas $(\mathrm{T}, \psi)$ : It may evaluate to null $(\mathrm{T})$, but it can occur in a branch together with non-null values $(\psi)$. The (T-PAIR), (T-FsT), and (T-SND) rules type pairs and projections. The (T-PAIR) rule states that a pair is not null $(\varphi, \mathrm{T})$. The projection rules (T-FsT), (T-SND) require that a pair cannot evaluate to null $(\mathrm{F}, \varphi)$. The (T-ABs) and (T-APP) rules are straightforward. The (T-ABs) rule states that an abstraction is non-null. The (T-ApP) rule requires that the applied lambda abstraction must be non-null. The (T-LET) rule is the standard Hindley-Milner generalization rule. The rule states that if we can type $e_{1}$ as $\tau_{1}$ under the environment $\Gamma$ then we may generalize the type $\tau_{1}$ to a type scheme $\sigma$, and type $e_{2}$ under an extended environment with $x: \sigma$. The (T-ITE) rule requires that the condition is a non-null Boolean and that the two branches have the same type. 


\subsection{Type Checking and Pattern Matching}

We now present the type rule (T-Choose). In Section 4.7, we will discuss a strict extension of the system with rule (T-CHOOSE- $\star$ ), which allows relational nullable polymorphism.

Given the expression choose $\overline{e_{m}}\left\{P \Rightarrow \overline{e_{b}}\right\}$, where $\overline{e_{m}}$ is the vector of match expressions, $P$ is the pattern matrix, and $\overline{e_{b}}$ is the vector of body expressions, (T-CHоOsE) imposes three requirements:

- We require that for each column $j$, the match expression can be typed as in $\Gamma \vdash_{d} e_{m}^{j}: \tau_{j}$ ? $\left(\varphi_{j}, \psi_{j}\right)$ - all match expressions $e_{m}^{j}$ can have different types $\tau_{j}$ and nullity formulas $\left(\varphi_{j}, \psi_{j}\right)$.

- We also require that the body expression of each row $i$ can be typed in an extended environment, as in $\Gamma \cup \Gamma_{i} \vdash_{d} e_{b}^{i}: \tau$. Note that a choice is a similar dataflow merge point as an if-then-else expression. Here we require that all possible body expressions have the same type $\tau$. This restriction will be lifted later in (T-CHOOSE- $\star$ ). The body $e_{b}^{i}$ may use variables that are introduced (bound) in the pattern. So the extended context $\Gamma_{i}$ extracts all variables from the non-null patterns in row $P_{i}$, i.e. those for which $P_{i, j}$ is a variable $x_{j}$. Their proper type should be the same as the corresponding match expression $e_{m}^{j}: \pi_{j}$. We add the nullity assumption $\left(\chi_{i, j}, \mathrm{~T}\right)$, which captures that row $i$ is only chosen when $e_{m}^{j}$ is non-null (since $P_{i, j}$ requested a non-null value for $x_{j}$ ).

- Finally, we require that the pattern matrix $P$ must be exhaustive for the vectors $\bar{\varphi}$ and $\bar{\psi}$.

Pattern Matrix Exhaustiveness. Intuitively, $P$ is exhaustive, if it has a matching row for each possible value of the match expressions $\overline{e_{m}}$. We only have to consider their nullity: 0 (for the null value) and 1 (for any non-null value). We first define the possible nullity values of an expression $e: \pi ?(b, c)$, for Boolean values $b, c \in \mathcal{B}$. Recall that $b$ indicates that $e$ may be null and $c$ indicates that $e$ may be a non-null value. For a vector $\bar{e}: \bar{\pi} ?(\bar{b}, \bar{c})$, the possible nullity vectors are all combinations of the nullity values of its elements:

$$
\begin{aligned}
\operatorname{values}(\mathrm{T}, \mathrm{T})= & \{0,1\} \quad \operatorname{values}(\mathrm{T}, \mathrm{F})=\{0\} \quad \operatorname{values}(\mathrm{F}, \mathrm{T})=\{1\} \quad \operatorname{values}(\mathrm{F}, \mathrm{F})=\emptyset \\
& \operatorname{valuesRow}(\bar{b}, \bar{c})=\operatorname{values}\left(b_{1}, c_{1}\right) \times \cdots \times \operatorname{values}\left(b_{n}, c_{n}\right)
\end{aligned}
$$

In particular, note that if one of the match expressions $e_{i}$ has nullity $(\mathrm{F}, \mathrm{F})$, then the whole vector is uninhabited $(\emptyset)$. Next, we define which nullity values are covered by a single pattern, and by a row of patterns:

$$
\begin{gathered}
\operatorname{covers}(\text { null })=\{0\} \quad \operatorname{covers}(x)=\{1\} \quad \operatorname{covers}(\square)=\{0,1\} \\
\operatorname{coversRow}(\bar{p})=\operatorname{covers}\left(p_{1}\right) \times \cdots \times \operatorname{covers}\left(p_{n}\right)
\end{gathered}
$$

We can now finally state when a pattern matrix is exhaustive:

Definition 4.3 (Exhaustiveness). A pattern matrix $P$ is exhaustive for the nullity formulas $(\bar{\varphi}, \bar{\psi})$, if for all valuations $V$, and for all $\bar{a} \in$ valuesRow $(V(\bar{\varphi}), V(\bar{\psi}))$, there exists a row $P_{i}$ in $P$, such that $\bar{a} \in \operatorname{coversRow}\left(P_{i}\right)$.

Example 4.4. Let $e_{1}: \pi$ ? ( $\left.\mathrm{F}, \beta\right)$ and let $e_{2}: \pi$ ? (T, T). We check exhaustiveness of the expression:

$$
\text { choose }\left(e_{1}, e_{2}\right)\{\text { case }(\varpi, x) \Rightarrow \cdots \text {, case }(y, \text { null }) \Rightarrow \cdots\}
$$

Note that $e_{1}$ must be a non-null value at runtime and that $e_{2}$ will be either null or a non-null value. For $\beta=\mathrm{F}$, valuesRow is empty. For $\beta=\mathrm{T}$, valuesRow $((\mathrm{F}, \beta),(\mathrm{T}, \mathrm{T}))=\{1\} \times\{0,1\}=\{(1,0),(1,1)\}$. Pattern row $(\square, x)$ covers instances $\{(0,1),(1,1)\}$ and pattern row (y, null) covers the instance $\{(1,0)\}$. So the value vector $(1,0)$ is covered by the second row, and the value vector $(1,1)$ is covered by the first row. Thus the pattern matrix is exhaustive for these match expressions. 
Soundness. We now match the nullity formulas $(\varphi, \psi)$ derived by the type system with the actual nullity of the values. The following "Canonical Forms Lemma" is essentially proved by inspecting the last applied derivation rule.

Lemma 4.5. Let $v$ be a typeable value with $\Gamma \vdash_{D} v: \pi ?(\varphi, \psi)$. Then:

(1) If $v=$ null then $\varphi \equiv_{\mathbb{B}} T$.

(2) If $v \neq$ null, then $\psi \equiv_{\mathbb{B}} T$.

We can now prove that, when we get to a well-typed choose expression at runtime, the exhaustive pattern matrix will always have a matching row, so the choose cannot "get stuck":

Lemma 4.6. Let $\bar{v}, \bar{\varphi}$ and $\bar{\psi}$ be given. Assume $\Gamma \vdash_{D} v_{j}: \pi_{j} ?\left(\varphi_{j}, \psi_{j}\right)$ (for each $j$ ). Assume that pattern matrix $P$ is exhaustive for $(\bar{\varphi}, \bar{\psi})$. Then matchRow $\left(P_{i}, \bar{v}\right)$ must be defined for some row $i$ in $P$.

Proof. Assume $\Gamma \vdash_{D} v_{j}: \pi_{j} ?\left(\varphi_{j}, \psi_{j}\right)$. Define $a_{j}:=0$, if $v_{j}=$ null and $a_{j}:=1$, otherwise. We claim $\left(^{*}\right)$ that $\bar{a} \in \operatorname{ValuesRow}(V(\bar{\varphi}), V(\bar{\psi}))$ for all valuations $V$. Then, since $P$ is exhaustive, we obtain that there is a row $i$ such that $\bar{a} \in \operatorname{coversRow}\left(P_{i}\right)$, i.e. for each $j, a_{j} \in \operatorname{covers}\left(P_{i, j}\right)$. By comparing the definition of covers and matchPat, we see that this implies that matchPat $\left(P_{i, j}, v_{j}\right)$ is defined for each $j$. But then also matchRow $\left(P_{i}, \bar{v}\right)$ is defined.

Now we prove the claim $\left(^{*}\right)$ that $\bar{a} \in \operatorname{ValuesRow}(V(\bar{\varphi}), V(\bar{\psi}))$, i.e. $a_{j} \in \operatorname{Values}\left(V\left(\varphi_{j}\right), V\left(\psi_{j}\right)\right)$, for each $j$. If $a_{j}=0$ then $v_{j}=$ null and by Lemma 4.5, $\varphi_{j} \equiv_{\mathbb{B}} \mathrm{T}$, so $V\left(\varphi_{j}\right)=\mathrm{T}$. Similarly, if $a_{j}=1$ then $v_{j} \neq$ null and by Lemma $4.5, \psi_{j} \equiv_{\mathbb{B}} \mathrm{T}$, so $V\left(\psi_{j}\right)=\mathrm{T}$. In both cases, $a_{j} \in \operatorname{Values}\left(V\left(\varphi_{j}\right), V\left(\psi_{j}\right)\right)$ by the definition of Values.

We are now in the position to state soundness of our declarative type system. The following two theorems guarantee that well-typed expressions cannot "get stuck":

Theorem 4.7 (Progress). Suppose e is a closed, well-typed expression (that is, $\vdash_{d} e: \tau$ for some $\tau$ ). Then either $e$ is a value or else there is some expression $e^{\prime}$ such that $e \rightarrow e^{\prime}$.

Theorem 4.8 (Preservation). If $\Gamma \vdash_{d} e: \tau$ and $e \rightarrow e^{\prime}$ then $\Gamma r_{d} e^{\prime}: \tau$.

\subsection{Type Inference with Algorithm $\mathrm{W}$ and Boolean Unification}

We now turn to type inference. Figure 8 presents an extension to the classical Algorithm W. It derives judgements of the form $\Gamma \vdash_{w} e: \tau ; S$. The judgement should be read as: Given as input a context $\Gamma$ and an expression $e$, compute as output its most general type $\tau$ and a substitution to the type variables, such that $\Gamma S r_{d} e: \tau$. If $e$ is not typeable in an instance of $\Gamma$, then the algorithm should fail. This happens for instance when some unification problem fails.

The rules are syntax directed: for each expression it is clear which rule to use. The antecedents should be executed in sequential order (top to bottom). There are three types of antecedents:

- Recursive calls to the algorithm $\vdash_{w}$ and repeated recursive calls $\vdash_{w}^{*}$. For instance, $(\mathrm{W}-\mathrm{ABs})$ has a simple recursive call, and (W-PAIR) has a repeated recursive call. We write $\Gamma \vdash^{*}{ }_{w} \overline{e_{1}, e_{2}}: \overline{\tau_{1}, \tau_{2}} ; S$ as an abbreviation of the two calls $\Gamma \vdash_{w} e_{1}: \tau_{1}^{\prime} ; S_{1}$ and $\Gamma S_{1} \vdash_{w} e_{2}: \tau_{2}^{\prime} ; S_{2}$. The returned types are $\overline{\left(\tau_{1}, \tau_{2}\right)}:=\overline{\left(\tau_{1}^{\prime} S_{2}, \tau_{2}^{\prime}\right)}$ and the substitution is $S:=S_{1} S_{2}$. This can be generalized to three calls (W-ITE) or even an arbitrary number of calls (W-CHOOSE), resembling a fold-operation.

- The creation of fresh type variables (proper type variables and nullity variables). This is presented as a non-functional operation, but we implemented a functional version, where fresh variables are derived in a canonical manner from the call graph of Algorithm W.

- Unification problems of the form $S=\left(t_{1} \stackrel{?}{=} t_{2}\right)$. This denotes that substitution $S$ is the most general unifier of $t_{1}$ and $t_{2}$. If the terms cannot be unified, Algorithm $\mathrm{W}$ fails. Type unification proceeds by structural matching with occurs check, as in Martelli-Montanari [Martelli and 


$$
\begin{aligned}
& \Gamma \vdash_{w} e: \tau ; S \\
& \alpha, \beta \text { fresh } \\
& \overline{\Gamma \vdash_{w} \text { null }: \alpha ?(\mathrm{~T}, \beta) ; i d} \\
& \text { (W-NuLL) } \\
& \Gamma \vdash_{w} e: \tau ; S_{1} \\
& \alpha_{1}, \beta_{1}, \gamma_{1}, \alpha_{2}, \beta_{2}, \gamma_{2}, \beta \text { fresh } \\
& \frac{S_{2}=\left(\tau \stackrel{?}{=}\left(\left(\alpha_{1} ?\left(\beta_{1}, \gamma_{1}\right)\right) *\left(\alpha_{2} ?\left(\beta_{2}, \gamma_{2}\right)\right) ?(\mathrm{~F}, \beta)\right)\right)}{\Gamma \vdash_{w} F s t(e):\left(\alpha_{1} ?\left(\beta_{1}, \gamma_{1}\right)\right) S_{2} ; S_{1} S_{2}} \\
& \Gamma \vdash_{w} \operatorname{Snd}(e):\left(\alpha_{2} ?\left(\beta_{2}, \gamma_{2}\right)\right) S_{2} ; S_{1} S_{2} \\
& \text { typeOf }(c)=\iota \\
& \frac{\beta \text { fresh }}{\Gamma \vdash_{w} c: \iota ?(\beta, \mathrm{T}) ; i d} \\
& \frac{(x, \sigma) \in \Gamma \quad \tau=\operatorname{inst}(\sigma)}{\Gamma \vdash_{w} x: \tau ; i d} \\
& \Gamma \vdash_{w}^{*} \overline{e_{1}, e_{2}}: \overline{\tau_{1}, \tau_{2}} ; S \\
& \beta \text { fresh } \\
& \overline{\Gamma{ }_{w}\left(e_{1}, e_{2}\right): \tau_{1} * \tau_{2} ?(\beta, \mathrm{T}) ; S} \\
& \text { (W-PAIR) } \\
& \Gamma \vdash_{w}^{*} \overline{e_{1}, e_{2}}: \overline{\tau_{1}, \tau_{2}} ; S_{0} \\
& \alpha, \beta, \gamma, \beta_{1} \text { fresh } \\
& \frac{S_{1}=\left(\tau_{1} \stackrel{?}{=}\left(\tau_{2} \rightarrow(\alpha ?(\beta, \gamma)) ?\left(\mathrm{~F}, \beta_{1}\right)\right)\right)}{\Gamma r_{w} e_{1} e_{2}:(\alpha ?(\beta, \gamma)) S_{1} ; S_{0} S_{1}}(\mathrm{~W}-\mathrm{APP}) \\
& \alpha, \beta, \gamma, \beta_{1} \text { fresh } \\
& \frac{\Gamma, x:(\alpha ?(\beta, \gamma)) \vdash_{w} e: \tau ; S}{\Gamma \vdash_{w} \lambda x . e:(\alpha ?(\beta, \gamma)) S \rightarrow \tau ?\left(\beta_{1}, \mathrm{~T}\right) ; S}(\mathrm{~W}-\mathrm{ABS}) \\
& \operatorname{inst}(\forall \bar{\gamma} \cdot \tau)=\tau[\bar{\gamma}:=\bar{\beta}] \text { for fresh variables } \bar{\beta} \\
& \text { (W-Fst / W-SND) } \\
& \Gamma \vdash_{w}^{*} \overline{e_{1}, e_{2}, e_{3}}: \overline{\tau_{1}, \tau_{2}, \tau_{3}} ; S_{0} \\
& \beta \text { fresh } \\
& S_{1}=\left(\tau_{1} \stackrel{?}{=} \text { Bool ? }(\mathrm{F}, \beta)\right) \\
& \frac{S_{2}=\left(\tau_{2} S_{1} \stackrel{?}{=} \tau_{3} S_{1}\right)}{\Gamma \vdash_{w} \text { if } e_{1} \text { then } e_{2} \text { else } e_{3}: \tau_{2} S_{1} S_{2} ; S_{0} S_{1} S_{2}}(\text { W-ITE }) \\
& \Gamma \vdash_{w} e_{1}: \tau_{1} ; S_{1} \quad \sigma=\operatorname{gen}\left(\Gamma S_{1}, \tau_{1}\right) \\
& \frac{\Gamma S_{1}, x: \sigma \vdash_{w} e_{2}: \tau_{2} ; S_{2}}{\Gamma \vdash_{w} \text { let } x=e_{1} \text { in } e_{2}: \tau_{2} ; S_{1} S_{2}}(\text { W-LET }) \\
& \Gamma \vdash_{w}^{*} \overline{\beta_{m}}: \overline{\pi ?(\varphi, \psi)} ; S_{0} \\
& \overline{\beta_{i, j}} \text { fresh variables } \\
& \Gamma_{\text {ext }, i}=\left\{x_{j}: \pi_{j} ?\left(\beta_{i, j}, \mathrm{~T}\right) \mid P_{i, j}=x_{j}\right\} \\
& \left(\Gamma \cup \overline{\Gamma_{\text {ext }}}\right) S_{0} \vdash_{w}^{*} \overline{e_{b}}: \bar{\tau} ; S_{1} \\
& S_{2}=\left\{\tau_{i} \stackrel{?}{=} \tau_{i+1}|0 \leq i<| \overline{e_{b}} \mid\right\} \\
& \frac{S_{3}=\left(\text { MatrixFormula }(P, \vec{\varphi}, \vec{\psi}) S_{1} S_{2} \stackrel{?}{=} \mathrm{T}\right)}{\Gamma \vdash_{w} \text { choose } \overline{e_{m}}\left\{P \Rightarrow \overline{e_{b}}\right\}: \tau_{0} S_{2} S_{3} ; S_{0} S_{1} S_{2} S_{3}} \\
& \operatorname{gen}(\Gamma, \tau)=\forall \bar{\gamma} \cdot \tau \text {, where } \bar{\gamma}=\mathrm{ftv}(\tau) \backslash \mathrm{ftv}(\Gamma)
\end{aligned}
$$

Fig. 8. Type Rules of Algorithm W for $\lambda_{\text {null }}^{\text {rel }}$

Montanari 1982], until two Boolean formulas must be unified. For Boolean unification (Sec. 4.1) we use successive variable elimination [Martin and Nipkow 1989]. We refer to [Madsen and van de Pol 2020] for a detailed description of the combination.

All rules, except (W-Choose), extend the standard rules of Algorithm W by propagating the nullity information. Note how "arbitrary formulas" $(\varphi, \psi)$ in the conclusions of the T-rules are implemented by fresh type variables in the corresponding W-rules (e.g. in the constructors (WNulL), (W-Cst), (W-PAIR) and (W-ABs)). These variables can become instantiated by further calls to Algorithm W. On the other hand, checks for nullity in the T-rules are implemented by unifying formulas with $\mathrm{F}$ in the corresponding W-rules (e.g. in the destructors (W-ApP), (W-FsT), (W-SND), (W-ITE)). When the T-rules require equal types, the W-rules implement this by unification (e.g. in (W-App), (W-ITE) and (W-Choose)). Hence, Algorithm W does not need the equivalent of the (T-EQ)-rule. The (W-LET) rule implements the standard generalization of types.

There is a small implementation detail: for generalization, the formulas in the context must be simplified to eliminate non-essential variables. ${ }^{3}$

\footnotetext{
${ }^{3}$ For instance, $\beta \vee \neg \beta$ should be simplified to T, to avoid blocking the generalization of $\beta$, ultimately leading to a less general type. This can be achieved by representing Boolean formulas in canonical form, such as BDDs or Boolean Rings.
} 
The rule (W-Choose) requires extra explanation. First, note the definition of the extended contexts $\Gamma_{\text {ext }, i}$. Here $\left(\beta_{i, j}, \mathrm{~T}\right)$ encodes that a variable $x_{j}$ in a pattern $P_{i}$ matches a non-null value. The polymorphic fresh variable $\beta_{i, j}$ can become instantiated when unifying it with types from other branches. By a slight abuse of notation, the following $\vdash_{w}^{*}$ uses different contexts $\Gamma \cup \Gamma_{\text {ext }, i}$ in each iteration. Recall that substitution $S_{0}$ is accumulated and threaded through this computation, resulting in the final $S_{1}$.

Next, we explain how we can infer the proper nullity information. A crucial step is to define a Matrix Formula that expresses the exhaustiveness of the pattern matrix $P$. The strategy to generate this matrix formula is (1) to create an "abstract" pattern matrix from $P$; (2) to saturate (and minimize) the pattern matrix; and (3) to transform the saturated pattern matrix to a Boolean formula.

The abstract pattern matrix $Q=\operatorname{Abstract}(P)$ consists of values in $\{0,1, \square\}$, and is obtained from the pattern matrix $P$ by replacing all its pattern variables by a 1 . Given the typed match expressions $e_{m}^{j}: \pi_{j} ?\left(\varphi_{j}, \psi_{j}\right)$ and an abstract pattern matrix $Q$, we define a Boolean formula that expresses that $\overline{e_{m}}$ is guaranteed to match some row $i$ of $Q$. In words, expressions matching a 1 cannot be null $\left(\neg \varphi_{j}\right)$ and expressions matching a 0 cannot be non-null $\left(\neg \psi_{j}\right)$. We ensure that we don't impose unnecessary restrictions for uninhabited types $(\mathrm{F}, \mathrm{F})$ :

$$
\begin{aligned}
\operatorname{Inhabited}(\bar{\varphi}, \bar{\psi}) & =\bigwedge_{j}\left(\varphi_{j} \vee \psi_{j}\right) \\
\operatorname{matchesRow}\left(Q_{i}, \bar{\varphi}, \bar{\psi}\right) & =\bigwedge_{j}\left\{\neg \varphi_{j} \mid Q_{i j}=1\right\} \wedge \bigwedge_{j}\left\{\neg \psi_{j} \mid Q_{i j}=0\right\} \\
\operatorname{matchesMatrix}(Q, \bar{\varphi}, \bar{\psi}) & =\bigvee_{i}\left\{\operatorname{matchesRow}\left(Q_{i}, \bar{\varphi}, \bar{\psi}\right)\right\} \\
\text { MatrixFormula }(P, \bar{\varphi}, \bar{\psi}) & =\neg \operatorname{lnhabited}(\bar{\varphi}, \bar{\psi}) \vee \text { matchesMatrix }(\operatorname{Saturate}(\operatorname{Abstract}(P)), \bar{\varphi}, \bar{\psi})
\end{aligned}
$$

The main reason why saturation must be applied, is that matchesMatrix requires that the match expressions are covered by a single row in the matrix. However, this is too strong, since an exhaustive matrix only requires that each instance of these expressions is covered by some row. By combining information from multiple rows into a single row, the saturated matrix has the property that it covers the match expressions by a single row. Before defining the saturation procedure in detail, we motivate and explain it with an example.

Example 4.9. Consider the program fragment

$$
\text { choose (null, } 5 \text {, if } b \text { then } 17 \text { else null) }\{\text { case (null, } x, y) \Rightarrow \cdots \text {, case }(\varpi, z \text {, null) } \Rightarrow \cdots\}
$$

The match expressions (null, 5 , if $b$ then 17 else null) have most general nullity information ( $\left.\mathrm{T}, \beta_{1}\right)$, $\left(\beta_{2}, \mathrm{~T}\right)$ and $(\mathrm{T}, \mathrm{T})$, i.e. their nullity vectors are $\bar{\varphi}=\left(\mathrm{T}, \beta_{2}, \mathrm{~T}\right)$ and $\bar{\psi}=\left(\beta_{1}, \mathrm{~T}, \mathrm{~T}\right)$. Note that in this case, Inhabited $(\bar{\varphi}, \bar{\psi}) \equiv_{\mathbb{B}} \mathrm{T}$. The corresponding nullity vectors are $\{0\} \times\{1\} \times\{0,1\}=\{(0,1,0),(0,1,1)\}$.

We match $(\bar{\varphi}, \bar{\psi})$ against the abstract pattern matrix of the two cases, which is $Q^{1}$ on the left:

$$
Q^{1}=\left(\begin{array}{lll}
0 & 1 & 1 \\
\square & 1 & 0
\end{array}\right) \Rightarrow Q^{2}=\left(\begin{array}{ccc}
0 & 1 & 1 \\
\square & 1 & 0 \\
0 & 1 & \square
\end{array}\right) \Rightarrow Q^{3}=\left(\begin{array}{ccc}
\square & 1 & 0 \\
0 & 1 & \square
\end{array}\right)
$$

Note that the nullity vectors are not covered by a single row, although value $(0,1,0)$ is covered by the second row and $(0,1,1)$ is covered by the first row. We saturate this matrix by adding a third row, which combines the "shared information" of the original rows (see middle matrix $Q^{2}$ ). Note that both nullity vectors are now covered by the new row in the saturated matrix. Finally, we can simplify the pattern matrix, since the first row is subsumed by the new third row $\left(Q_{1}^{2}\right.$ covers a subset 
of the nullity vectors covered by $Q_{3}^{2}$ ). This results in the matrix $Q^{3}$. Simplification is not obligatory, but it helps in reducing the final formula. For this example, the generated Matrix Formula is:

$$
\text { matchesRow }\left(Q_{1}^{3}, \bar{\varphi}, \bar{\psi}\right) \vee \text { matchesRow }\left(Q_{2}^{3}, \bar{\varphi}, \bar{\psi}\right)=\left(\neg \beta_{2} \wedge \neg \mathrm{T}\right) \vee\left(\neg \beta_{1} \wedge \neg \beta_{2}\right) \equiv_{\mathbb{B}} \neg \beta_{1} \wedge \neg \beta_{2}
$$

Algorithm $\mathrm{W}$ unifies $\neg \beta_{1} \wedge \neg \beta_{2} \stackrel{?}{=} \mathrm{T}$, yielding the most general substitution $\left\{\beta_{1} \mapsto \mathrm{F}, \beta_{2} \mapsto \mathrm{F}\right\}$.

We now define the saturation and simplification procedure more formally. Define a partial order on abstract patterns $\{0,1, \odot\}$ by the following laws:

$$
0 \leq \boxminus \quad 1 \leq \boxminus \quad x \leq x
$$

The corresponding greatest lowerbound on elements is defined by the following laws:

$$
x \sqcap \boxminus=x \quad \square \sqcap x=x \quad x \sqcap x=x
$$

Note that $0 \sqcap 1$ and $1 \sqcap 0$ are undefined, since 0 and 1 have no common lowerbound in this partial order. Next, extend this partial order and the partial greatest lowerbound function in a pointwise fashion to vectors; in particular, we will apply them to rows in the pattern matrix.

Define the partial operation $\oplus$ to combine compatible rows as follows:

$$
\left(\overline{x_{0}}, 1, \overline{x_{1}}\right) \oplus\left(\overline{y_{0}}, 0, \overline{y_{1}}\right)=\left(\overline{z_{0}}, \varpi, \overline{z_{1}}\right) \text {, iff } \overline{z_{0}}:=\overline{x_{0}} \sqcap \overline{y_{0}} \text { and } \overline{z_{1}}:=\overline{x_{1}} \sqcap \overline{y_{1}} \text { are defined. }
$$

Example 4.10. In Example 4.9, $Q_{1}^{2} \leq Q_{3}^{2}$ since in the third column, $1 \leq \varpi$. The third row in $Q^{2}$ is explained by $(0,1,1) \oplus(\varpi, 1,0)=(0,1, \varpi)$, with $\overline{x_{0}}=(0,1), \overline{y_{0}}=(\varpi, 1)$, and $\overline{z_{0}}=(0,1) \sqcap(\varpi, 1)=(0,1)$. In this example, the rightmost vectors $\overline{x_{1}}=\overline{y_{1}}=\overline{z_{1}}=()$.

Definition 4.11 (Saturated and Minimal). An abstract pattern matrix $Q$ is saturated, if for every two rows $Q_{i}$ and $Q_{j}$, if $Q_{i} \oplus Q_{j}$ is defined, then there is a row $Q_{k}$ with $Q_{i} \oplus Q_{j} \leq Q_{k}$.

An abstract pattern matrix $Q$ is minimal, if for no two different rows $Q_{i}$ and $Q_{j}, Q_{i} \leq Q_{j}$.

LEMma 4.12. For every abstract pattern matrix $Q$, there exists a unique (modulo order of rows) saturated and minimal matrix Saturate $(Q)$.

Proof. We first saturate the matrix, by repeatedly applying saturation steps: While $Q_{i}$ and $Q_{j}$ are rows in the matrix and $Q_{i} \oplus Q_{j}$ is defined but not a row in the matrix, then add the row $Q_{i} \oplus Q_{j}$. Since we only add rows, and the number of possible different rows is finite, this process terminates with a unique result (modulo the order of rows) and yields a saturated matrix.

Next, we simplify the matrix by minimization steps: While $Q_{i}$ and $Q_{j}$ are different rows with $Q_{i} \leq Q_{j}$, remove row $Q_{i}$. Since we only remove rows, this process terminates with a unique result: the set of minimal rows, hence it yields a minimal matrix. Note that every minimization step keeps the matrix saturated (since $Q_{j}$ still covers everything covered by $Q_{i}$ ).

So the result is uniquely defined (modulo row order), minimal, and saturated.

In Example 4.9, $Q^{1} \Rightarrow Q^{2}$ was a saturation step and $Q^{2} \Rightarrow Q^{3}$ was a minimization step. ${ }^{4}$

LEMMA 4.13. Let $Q$ be an abstract pattern matrix.

(1) Saturate $(Q)$ covers the same nullity vectors as $Q$;

(2) If $Q$ is saturated and covers all nullity vectors in valuesRow $(\bar{b}, \bar{c})$, then these are already covered by a single row $Q_{i}$;

(3) "P is exhaustive for $\bar{\varphi}, \bar{\psi} " \equiv_{\mathbb{B}}$ MatrixFormula $(P, \bar{\varphi}, \bar{\psi})$.

Proof. (1) In saturation, we add a row $Q_{i} \oplus Q_{j}$, but all its instances are already covered by either $Q_{i}$ or by $Q_{j}$. In minimization, we remove row $Q_{i}$, but all its instances are also covered by $Q_{j}$.

\footnotetext{
${ }^{4}$ In the implementation, we minimize the matrix initially and after each saturation step, and we only add $Q_{i} \oplus Q_{j}$ if it is not yet covered by any $Q_{k}$, in order to avoid adding rows that would be removed later.
} 
(2) Let $\overline{a_{1}}, \overline{a_{2}} \in \operatorname{ValuesRow}(\bar{b}, \bar{c})$. Let $A_{i}:=\left\{a_{1}^{i}, a_{2}^{i}\right\}$, then for each $i, A_{i} \subseteq \operatorname{Values}\left(b_{i}, c_{i}\right)$, by definition of ValuesRow. By assumption, all elements in $A_{1} \times \cdots \times A_{n}$ are covered by $Q$. Define $a_{3}^{i}=a_{1}^{i}$, if $a_{1}^{i}=a_{2}^{i}$, and $a_{3}^{i}=\square$, otherwise. Since $Q$ is saturated, $\overline{a_{3}}$ is covered by some row $k$ of $Q$. But then $\overline{a_{1}}$ and $\overline{a_{2}}$ are covered by the same row $k$ in $Q$.

(3) By Lemma 4.12, we can compute $Q=\operatorname{Saturate}(\operatorname{Abstract}(P))$.

$\Rightarrow$ : Let $P$ be exhaustive. There are two cases. If $(\bar{\varphi}, \bar{\psi})$ contains a $(\mathrm{F}, \mathrm{F})$ entry for all valuations, then $\neg$ Inhabited $(\bar{\varphi}, \bar{\psi})$ holds, and the Matrix Formula is true.

Otherwise, there is a valuation $V$ such that valuesRow $(V(\bar{\varphi}), V(\bar{\psi})) \neq \emptyset$. Since $P$ is exhaustive, all $\bar{a}$ in its nullity vector are covered by $P$. By (1), $Q$ still covers the same $\bar{a}$. By (2), they are even covered by a single row $Q_{k}$. Hence matchesRow $\left(Q_{k}, V(\bar{\varphi}), V(\bar{\psi})\right)$ holds, and the Matrix Formula is true.

$\Leftarrow$ : Assume $V$ (MatrixFormula $(P, \bar{\varphi}, \bar{\psi}))=\mathrm{T}$ for arbitrary $V$. We must show that $P$ is exhaustive for $(V(\bar{\varphi}), V(\bar{\psi}))$. Let $\bar{a} \in$ valuesRow $(V(\bar{\varphi}), V(\bar{\psi}))$ be given, then Inhabited $(V(\bar{\varphi}), V(\bar{\psi}))$ is true, so matchesMatrix $(Q, V(\bar{\varphi}), V(\bar{\psi}))$ holds. So for some row $k$, the disjunct matchesRow $\left(Q_{k}, V(\bar{\varphi}), V(\bar{\psi})\right)$ holds. This means that $Q_{k}$ covers $\bar{a}$, hence $Q$ covers $\bar{a}$, hence $P$ covers $\bar{a}$ by (1). Hence $P$ is exhaustive.

Example 4.14. In Example 4.4, we obtained the pattern matrix $P=((๑, 1),(1,0))$. We saturate it by adding the generalized row $(1, \bullet)$. Now we remove the redundant row $(1,0)$. So the minimal saturated pattern matrix is $((\bullet, 1),(1, \odot))$. Recall that the nullity of the matched terms was $(F, \beta)$ and $(T, T)$. So the Matrix Formula in this case is: $(\neg T \vee \neg F) \equiv_{\mathbb{B}} T$. Indeed, the matrix is exhaustive, since the given expressions are covered by the second row of the minimal saturated matrix.

Lemma 4.13(3) is the key to relate the declarative system with Algorithm W. We formulate the correctness of Algorithm W with null and pattern matching as follows:

Theorem 4.15 (Soundness of W). If $\Gamma \vdash_{w} e: \tau ; S$ then $\Gamma S \vdash_{d} e: \tau$.

Theorem 4.16 (Completeness of W). If $\Gamma S \vdash_{d} e: \tau$ then for some $\tau^{\prime}$ and $S^{\prime}, \Gamma \vdash_{w} e: \tau^{\prime} ; S^{\prime}$ and there exists $S_{0}, \tau_{0}$ such that $\Gamma S^{\prime} S_{0}=\Gamma S$ and gen $\left(\Gamma S^{\prime}, \tau^{\prime}\right) S_{0} \sqsubseteq \tau_{0} \equiv_{\mathbb{B}} \tau$.

Corollary 4.17 (Principal Types). The calculus $\lambda_{\text {null }}^{\text {rel }}$ enjoys the principal type property.

\subsection{Extension: Polymorphic Relational Nullability with the CHOose- $\star$ Rule}

We now discuss an extension of the type system that enables relational polymorphic nullability. Consider the following two program fragments:

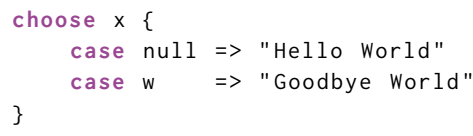

The left program fragment can be given the non-nullable type String ? (F, T), because both branches return a non-null string. On the other hand, the program fragment on the right cannot be given the non-nullable type Int ? $(\mathrm{F}, \mathrm{T})$ - not even when $x$ would be a constant value. The reason is that the (T-Choose) rule, like (T-ITE), requires that the types of all branches must be the same, which would be Int ? $(\mathrm{T}, \mathrm{T})$ in this case. Thus, even if we know that $x$ cannot possibly be null, this information is not preserved in the type of a choose expression. In our extension, the nullities of the branches will be combined with $\vee$. Moreover, the type of each branch will depend on its pattern. Consequently, the program fragment on the right gets type Int ? $(\beta, \gamma)$, whenever $x:$ Int ? $(\beta, \gamma)$. This is achieved by the (CHOose- $\star$ ) rule, shown in Figure 9. A similar extension can be made to the (T-ITE) rule.

4.7.1 Typing. In order to infer a more precise, relational nullable type, we now extend the declarative type system with (T-CноOSE- $\star$ ) and Algorithm W with (W-CHOOSE- $\star$ ), see Figure 9. The old rules (T-Choose) and (W-Choose) are now superfluous. 


$$
\begin{aligned}
& \Gamma \vdash_{d} e_{m}^{j}: \pi_{j} ?\left(\varphi_{j}, \psi_{j}\right) \quad(\forall j) \\
& \Gamma_{\text {ext }, i}:=\left\{x_{j}: \pi_{j} ?\left(\chi_{i, j}, \mathrm{~T}\right) \mid P_{i, j}=x_{j}\right\} \\
& \Gamma \cup \Gamma_{\text {ext }, i} \vdash_{d} e_{b}^{i}: \pi^{\prime} ?\left(\varphi_{i}^{\prime}, \psi_{i}^{\prime}\right) \quad(\forall i) \\
& P \text { is exhaustive for }(\bar{\varphi}, \bar{\psi}) \\
& \varphi_{r}=\bigvee_{i}\left(\operatorname{Line}\left(P_{i}, \bar{\varphi}, \bar{\psi}\right) \wedge \varphi_{i}^{\prime}\right) \\
& \psi_{r}=\bigvee_{i}\left(\text { Line }\left(P_{i}, \bar{\varphi}, \bar{\psi}\right) \wedge \psi_{i}^{\prime}\right) \\
& \overline{\Gamma r_{d} \text { choose } \overline{e_{m}}\left\{P \Rightarrow \overline{e_{b}}\right\}: \pi^{\prime} ?\left(\varphi_{r}, \psi_{r}\right)} \\
& \text { (T-CHOOSE- } \star \text { ) }
\end{aligned}
$$

$$
\begin{gathered}
\Gamma \vdash_{w}^{*} \overline{\beta_{m}}: \overline{\pi ?(\varphi, \psi)} ; S_{0} \\
\beta_{i, j} \text { fresh variables } \\
\Gamma_{\text {ext }, i}:=\left\{x_{j}: \pi_{j} ?\left(\beta_{i, j}, \mathrm{~T}\right) \mid P_{i, j}=x_{j}\right\} \\
\left(\Gamma \cup \overline{\Gamma_{\text {ext }}}\right) S_{0} \vdash_{w}^{*} \overline{e_{b}}: \overline{\pi^{\prime} ?\left(\varphi^{\prime}, \psi^{\prime}\right)} ; S_{1} \\
S_{2}=\left\{\pi_{i}^{\prime} \stackrel{?}{=} \pi_{i+1}^{\prime}|0 \leq i<| \overline{e_{b}} \mid\right\} \\
S_{3}=\left(\text { MatrixFormula }(P, \bar{\varphi}, \bar{\psi}) S_{1} S_{2} \stackrel{?}{=} \mathrm{T}\right) \\
\varphi_{r}=\bigvee_{i}\left(\operatorname{Line}\left(P_{i}, \bar{\varphi}, \bar{\psi}\right) S_{1} \wedge \varphi_{i}^{\prime}\right) \\
\psi_{r}=\bigvee_{i}\left(\operatorname{Line}\left(P_{i}, \bar{\varphi}, \bar{\psi}\right) S_{1} \wedge \psi_{i}^{\prime}\right) \\
\hline \Gamma \vdash_{w} \text { choose } \overline{e_{m}}\left\{P \Rightarrow \overline{e_{b}}\right\}: \\
\left(\pi_{0}^{\prime} ?\left(\varphi_{r}, \psi_{r}\right)\right) S_{2} S_{3} ; S_{0} S_{1} S_{2} S_{3}
\end{gathered}
$$

(W-CHOose- $\star$ )

Fig. 9. Extension of Declarative Type System and Algorithm W with CHOosE- $\star$

In both cases, we still require that the proper types $\pi^{\prime}$ of all the bodies are equal. However, we now allow different nullity formulas for the various branches. The nullity formulas of the whole choose-construct will be the disjunction of the formulas of the branches. In order to specify relational nullable types, we strengthen the result types of the branch $i$ with the information that the corresponding pattern row has matched. This information is encoded as the formula $\operatorname{Line}\left(P_{i}, \bar{\varphi}, \bar{\psi}\right)$. A row matches, if each single entry $j$ matches an expression with nullity formula $\left(\varphi_{j}, \psi_{j}\right)$, which is encoded in the formula mayCover $\left(P_{i, j}, \varphi_{j}, \psi_{j}\right)$. Pattern null can only apply if the expression may be null, i.e., $\varphi$ holds. Pattern $x$ can only apply if the expression may be non-null, i.e. $\psi$ holds. Finally, pattern $\square$ can only apply if the expression is either null or non-null, i.e. $\varphi \vee \psi$ holds.

$$
\text { mayCover }(\text { null, } \varphi, \psi)=\varphi \quad \operatorname{may} \operatorname{Cover}(x, \varphi, \psi)=\psi \quad \operatorname{mayCover}(\varpi, \varphi, \psi)=\varphi \vee \psi
$$

Row $i$ may apply, if all its entries may cover the corresponding match value:

$$
\operatorname{Line}\left(P_{i}, \bar{\varphi}, \bar{\psi}\right)=\bigwedge_{j}\left(\operatorname{may} \operatorname{Cover}\left(P_{i, j}, \varphi_{j}, \psi_{j}\right)\right)
$$

4.7.2 Soundness. We would like to state soundness, i.e. the progress and preservation theorems, for the declarative type system extended with the (T-CHOose- $\star$ ) rule. Progress is straightforward, but it turns out that preservation does not hold. To understand why, consider the following program fragment which is typeable with (T-CHоose- $\star$ ) but not with (T-CHоose):

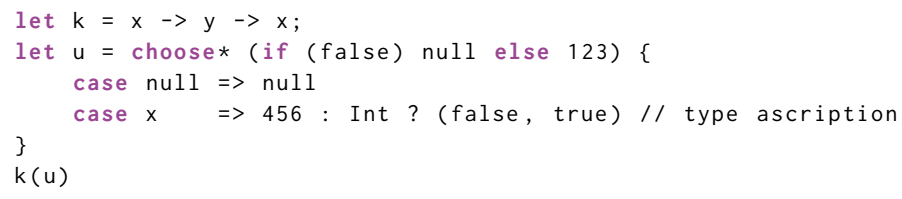

Here the proper type of $k(u)$ is $\alpha \rightarrow \operatorname{Int} 32$ ? (T, T) for some $\alpha$. This type arises because the type of the if-then-else expression admits both null and non-null values and, consequently, the type system cannot exclude either branch of the choose expression and thus $u$ may be null or non-null.

Let us now perform two reduction steps: First, the if-then-else expression is reduced to the value 123. Next, the choose expression is reduced to the value 456 (which is given the explicit type Int ? $(\mathrm{F}, \mathrm{T}))$ with a type ascription ${ }^{5}$. After these two reduction steps, the proper type of $k(u)$ is $\alpha \rightarrow \operatorname{Int} 32$ ? (F,T). But the two proper types: $\alpha \rightarrow \operatorname{Int} 32$ ? (T, T) and $\alpha \rightarrow \operatorname{lnt} 32$ ? (F, T) are

\footnotetext{
${ }^{5}$ The use of a type ascription is immaterial, the exact same typing can be obtained with an even more elaborate program.
} 
not equal - not even modulo Boolean equivalence. Consequently, in the declarative type system extended with the (T-CHOоse- $\star$ ) rule, preservation does not hold in its original formulation.

However, all is not lost. We conjecture that preservation still holds if the type system is extended with a notion of "Boolean sub-typing". Informally, $\tau<: \tau^{\prime}$ if $\tau$ is structurally equivalent to $\tau^{\prime}$ and whenever there are two Boolean formulas $\varphi$ and $\varphi^{\prime}$ then $\varphi \Rightarrow \varphi^{\prime}$ (or $\varphi^{\prime} \Rightarrow \varphi$ in contravariant positions). With this notion, we believe:

Conjecture 4.18 (Preservation- $\star$ ). If $\Gamma \vdash_{d} e: \tau$ and $e \rightarrow e^{\prime}$ then $\Gamma r_{d} e^{\prime}: \tau^{\prime}$ for some $\tau^{\prime}<: \tau$.

However, Boolean sub-typing, with the corresponding extension of principal type inference, deserves its own separate treatment, which is left as future work.

\section{IMPLEMENTATION}

We have implemented the $\lambda_{\text {null }}^{\text {rel }}$ calculus in two systems: As a minimal proof-of-concept calculus and as an extension of the Flix programming language.

\subsection{The Flix Programming Language}

Flix is a functional, imperative, and logic programming language that supports algebraic data types, pattern matching, parametric polymorphism, type classes, currying, higher-order functions, polymorphic effects, extensible records, first-class Datalog constraints, channel and process-based concurrency, and tail call elimination [Madsen and Lhoták 2018, 2020; Madsen and van de Pol 2020; Madsen et al. 2016]. The Flix compiler project, including the standard library and tests, is approximately 135,000 lines of Flix and Scala code.

\subsection{Minimal Proof-of-Concept Implementation}

We have implemented a minimal proof-of-concept version of the $\lambda_{\text {null }}^{\text {rel }}$ calculus as a Flix program. The proof-of-concept includes a complete implementation of the type inference system based on Algorithm W. The implementation closely follows the formal type inference rules (Section 4.6).

\subsection{Flix Extension with Relational Nullable Types}

Flix, like Haskell, OCaml, and Rust does not have a null value, but instead has the Option data type. However, as discussed in Section 2, relational nullable types are also relevant for programming languages with Option types. For Flix, we introduce a new data type called Choice[t, a, p] which is parameterized by a type $t$, and two booleans a and $p$. The Choice data type has two constructors:

$$
\text { Absent : Choice[t, true, } p] \quad \text { Present : Choice[t, a, true] }
$$

Note how the type of Absent and Present closely mirrors that of null and non-null values.

To work with Choice values, we introduce two pattern matching constructs: choose and choose* corresponding to the type rules (T-ChOose) and (T-CHOose- $\star$ ). A choose expression destructs a sequence of choice values into a value of any type, whereas a choose* expression destructs a sequence of choice values into a value that must be of the Choice type. Other than these two changes, everything else follows the formal system.

In terms of implementation effort, the extension required less than 3,000 lines of code, mostly related to computing saturated pattern match matrices and type inference for the choose and

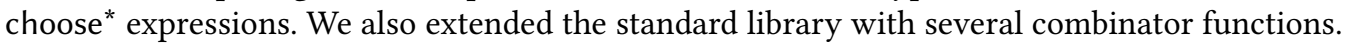

Flix, with our extension, is open source, ready for use, and freely available at:

https://flix.dev/ and https://github.com/flix/flix/ 
5.3.1 Higher-Order Combinators. The extended Flix type system naturally supports combinator functions such as filter, map, flatMap, and flatten. ${ }^{6}$

For example, the definition of map is straightforward:

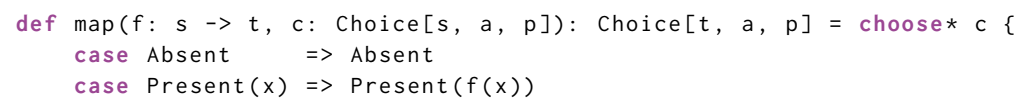

The definition of flatMap is also straightforward, but gives rise to a more interesting type:

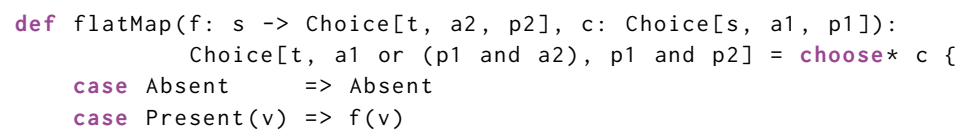

Let us break it down. Let us consider when the result can be Absent. This can happen for two reasons: If the argument $c$ is already Absent (i.e. $a_{1}$ holds) or if $f$ returns Absent (i.e. $a_{2}$ holds) and the argument $c$ was Present (i.e. $p_{1}$ holds). This gives us the constraint $a_{1} \vee\left(p_{1} \wedge a_{2}\right)$. Now, let us consider when the result can be Present. This can only happen if the argument $c$ is already Present (i.e. $p_{1}$ holds) and $f$ returns a Present value (i.e. $p_{2}$ holds). This gives us the constraint $p_{1} \wedge p_{2}$.

The definition of filter is also straightforward:

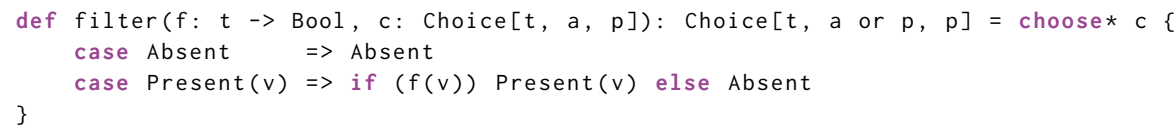

Let us break it down again. First, observe that we have no information about whether $f$ returns true or false, consequently in the Present case the value can be Absent or Present. Let us now consider when the result can be Absent. This can happen for two reasons: If the argument $c$ is already Absent (i.e. $a$ holds) or if $c$ is Present (i.e. $p$ holds). This gives us the constraint $a \vee p$. Let us consider when the result can be Present. This can only happen if $c$ is Present (i.e. if $p$ holds).

We can define a withDefault function that, given two arguments, returns the first argument if it is Present and otherwise returns the second argument:

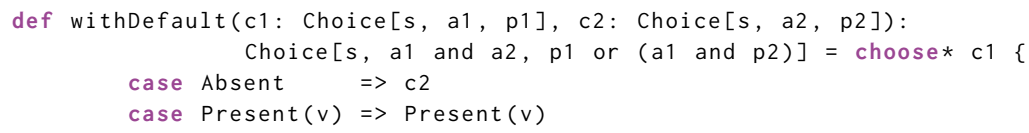

Let us break it down again. Let us consider when the result can be Absent. This can only happen if $c_{1}$ is Absent (i.e. $a_{1}$ holds) and if $c_{2}$ is also Absent (i.e. $a_{2}$ holds). Thus the result can be Absent when $a_{1} \wedge a_{2}$ holds. Let us now consider when the result can be Present. This can happen if $c_{1}$ is Present (i.e. $p_{1}$ holds) or if $c_{1}$ is Absent and $c_{2}$ is Present (i.e. $a_{1}$ holds and $p_{2}$ holds). Thus the result can be Present when $p_{1} \vee\left(a_{1} \wedge p_{2}\right)$ holds.

We close with an interesting, if perhaps useless, combinator:

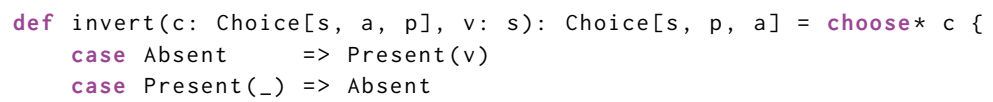

For example, invert (invert (invert(Absent, 1), 2), 3) $==3$. To the best of our knowledge, no existing nullable type system can express such a combinator.

\footnotetext{
${ }^{6}$ For the sake of exposition, we have slightly simplified some of the type schemes. The type schemes are still correct, but
} they are less general than the most general type scheme permitted by the relational nullable type system. 
5.3.2 Type Errors. An important practical concern is how to report type errors to the programmer. In particular, Hindley-Milner style type inference is known to produce type errors that are difficult for programmers to understand [Chitil 2001]. The use of Boolean formulas and unification only exacerbates this problem.

In the current Flix implementation, we report a type error when two Boolean formulas $\varphi$ and $\psi$ fail to unify. We have found that such type errors are difficult to untangle. In future work, we would like to explore a better approach: in case of a unification failure, we want to construct a sequence of nullable or non-nullable values that are not matched by any row in the pattern match. We think that such type errors could be more helpful than ordinary unification errors.

\subsection{Evaluation: Recasting the Program Fragments in Flix}

We have used the Flix implementation to experiment with reformulations of the program fragments from the preliminary study (Section 2). We believe that all program fragments can be suitably reformulated in Flix using the relational nullable type system. To illustrate, we show three examples from the study recast in Flix:

\section{pmd/pmd (Recast in Flix)}

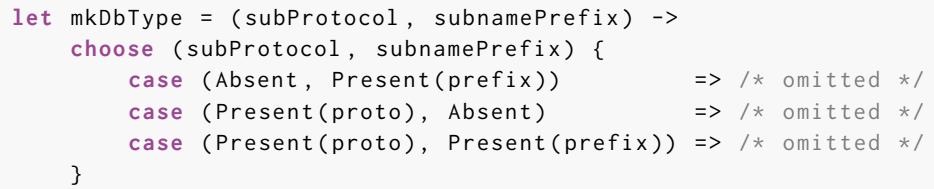

which captures that subProtocol and subnamePrefix cannot both be Absent.

openssl/openssl (Recast in Flix)

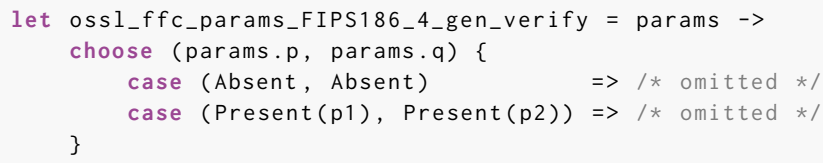

which captures that the $\mathrm{p}$ and $\mathrm{q}$ fields of the record params must both be Absent or Present.

snowplow/iglu (Recast in Flix)

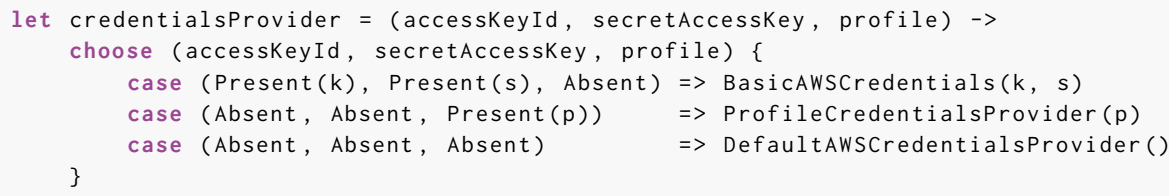

which captures that either both accessKeyld and secretAccessKey must be provided, or profile must be provided, or none of the arguments must be provided.

All three examples illustrate the need for relational nullability and the need for may and must information, i.e., whether an expression may or must evaluate to null and whether it may or must evaluate to a non-null value. 


\section{RELATED WORK}

\subsection{Boolean Unification}

Early work on Boolean unification and the successive variable elimination algorithm goes back to George Boole himself [Boole 1847]. Later work include that of Rudeanu [1974] and Buttner and Simonis [1987]. An accessible introduction to Boolean unification is provided by Martin and Nipkow [1989]. Boudet et al. [1989] study unification in Boolean rings and in combination with other theories. A study of the computational complexity of Boolean unification is provided by Baader [1998]. An alternative algorithm for Boolean unification is proposed by Löwenheim [1908].

\subsection{Type Systems and Type Inference}

The Damas-Hindley-Milner type system was first described by Hindley [1969] and Milner [1978]. Later, Damas [1984] studied the formal foundations of the system. The Damas-Hindley-Milner type system has been used as the theoretical foundation for several real-world functional programming languages, including Haskell, OCaml, and Standard ML. Many extensions have been proposed, notably type classes [Wadler and Blott 1989], qualified-types [Jones 2003], and region-based memory management [Tofte and Talpin 1997].

In the early years, an important question was how to prove correctness of Damas-HindleyMilner-style type systems and similar systems. In an influential work, Wright and Felleisen [1994] describes a "syntactic approach" laying the foundations for the modern formulations of soundness in terms of the progress and preservation theorems.

\subsection{Type Inference with Boolean Unification}

In [Madsen and van de Pol 2020], we propose a polymorphic type and effect system based on Hindley-Milner and Boolean unification. In that system, every expression is associated with a Boolean formula that captures when the expression is pure (i.e. has no side-effect). The type and effect system supports effect polymorphism: The purity of a higher-order function may depend on the purity of its function arguments. The authors implement the type and effect system in the Flix programming language. Experimental results on the Flix standard library and a number of Flix applications suggest that the performance cost of Boolean unification during type and effect inference is acceptable.

While our work in [Madsen and van de Pol 2020] and this paper are both based on the HindleyMilner type system extended with Boolean constraints, there are significant differences: First, the subject areas are very different: effect polymorphism vs. relational nullability. In [Madsen and van de Pol 2020], the goal is to capture when an expression is pure, whereas here our goal is to capture the nullability of an expression in relation to other related expressions and to express the exhaustiveness condition of the choose construct in the type system. Second, in [Madsen and van de Pol 2020], the Boolean formulas have no impact on the progress theorem (ill-effected expressions cannot get stuck). Third, our current type system relies on both may and must information.

\subsection{Nullable Type Systems}

There is a rich research literature on nullable type systems. We aim to provide a broad outline. Most of the work below is orthogonal to the idea of relational nullability.

6.4.1 Studies. Chalin and James [2007] conduct at study of five large open source projects. The study shows that on average $3 / 4$ of all references could be annotated as non-null. Consequently, the authors proposed that non-nullable types should be the default. In relation to our work, null or non-null annotations are never required since our type system supports type inference; nullness is simply inferred from the source code. 
6.4.2 Retrofitted Nullable Type Systems. Fähndrich and Leino [2003] propose a sound way to retrofit C\# and Java with nullable type systems. Nieto et al. [2020b] present an extension of the Scala type system that makes nullable types explicit. The type system has been implemented in the Dotty compiler for the Scala 3.0 language. Our "retrofit" of Flix was straightforward since we simply introduced a new data type called Choice. An interesting question for future work would be to explore how to retrofit relational nullable type system onto other programming languages.

6.4.3 Gradual Nullable Type Systems. Brotherston et al. [2017] present Granullar, a gradual pluggable type system for Java. The type system ensures that NullPointerExceptions cannot occur within checked code; only on the boundary between checked and unchecked code. Nieto et al. [2020a] present a blame calculus for a gradually typed language with null.

6.4.4 Object Initialization. Fähndrich and Xia [2007] present a type system with delayed types that allows reasoning about when an object becomes fully initialized. Qi and Myers [2009] present a type system based on type state that prevents reading from uninitialized fields. The calculus side-steps the issue of null by relying on a flow-sensitive type system to track the set of uninitialized fields Summers and Müller [2011] presents a type system to track object initialization. The type system ensures that objects under construction, whose invariants may not yet be fully satisfied, cannot escape except under controlled circumstances. While beyond the scope of this paper, relational nullability seems related to the problem of object initialization in the sense that whether one field is initialized may depend on whether another field is initialized. It would be interesting to explore this connection in future work.

6.4.5 Static Analysis. Spoto [2008] presents a static analysis to detect NullPointerExceptions in Java bytecode. The static analysis uses abstract domains implemented efficiently using binary decision diagrams (BDDs). While their work and our work both use Boolean formulas, the two approaches are very different. For future work, it would be interesting to explore whether the idea of relational nullability can be formulated as a (relational) abstract domain. Male et al. [2008] present an extension of the JVM bytecode verifier with support for nullable types. Hubert et al [2008] present a constraint-based static analysis to infer non-null annotations. Banerjee et al. [2019] presents NullAway, a tool to find NullPointerExceptions . NullAway, unlike other similar tools, does not aim for soundness, but rather for a reduction of spurious warnings reported by the tool. This reduces the annotation burden for the programmer. Our type system supports type inference, so annotations are never required.

6.4.6 Unsoundness. Amin and Tate [2016] demonstrate unsoundness of Java's and Scala's type systems. The unsoundness manifests itself as a combination of wildcards, sub-typing, and nulls. Intriguingly, each individual feature is believed to be sound, but it is their combination that breaks soundness of the overall type systems.

\subsection{More Powerful Type Systems}

6.5.1 Logical Types. Tobin-Hochstadt and Felleisen [2010] present a type system with logic predicates for a typed Scheme. The key idea is to use control flow predicates as propositional logic formulas as part of the type of an expression. For example, in the Scheme expression (if (number? $\mathrm{x})$ e $1 \mathrm{e} 2$ ), the $e_{1}$ expression is typed with the knowledge that $x$ satisfies the number? predicate, whereas $e_{2}$ is typed with the knowledge that $x$ does not satisfy the predicate. Concretely, in their system, the typing judgement is of the form $\Gamma \vdash \varphi_{+} \mid \varphi_{-} ; o$ which states that if $e$ evaluates to a "truthy" value then the $\varphi_{+}$formula holds (and otherwise the $\varphi_{-}$formula holds).

The work of Tobin-Hochstadt and Felleisen is closely related to ours, but with several differences. First, we introduce the notion of relational nullability and we compute a precise exhaustiveness 
condition. Second, our type system supports parametric polymorphism whereas their type system supports sub-typing (neither type system supports both). Third, our type system supports full type inference (i.e. if a program is typeable w.r.t. the declarative system then Algorithm W will provide the typing), whereas their type system supports a limited form of local type inference [Pierce and Turner 2000].

6.5.2 Refinement Types. Our relational nullable type system can be seen as a special case of a refinement type system [Rondon et al. 2008; Vazou et al. 2014], which has typing judgements of the form $\Gamma \vdash e:\{v: \tau \mid \varphi\}$. Here $\tau$ is the type of the expression $e$ subject to the logic formula $\varphi$, which may refer to the value of $e$ as $v$. For example, the refinement type $\{v: \operatorname{lnt} \mid 1 \leq v \leq 99\}$ captures all integers between 1 and 99 (inclusively). With this in mind, we can view the relational nullable type $\pi ?(\varphi, \psi)$ as the refinement type $\{v: \pi \mid v=$ null $\Rightarrow \varphi \wedge v \neq$ null $\Rightarrow \psi\}$.

Refinement type systems are often very powerful depending on the types of formulas that are admitted. If such formulas contain undecidable fragments of logic then type checking may even be undecidable [Rondon et al. 2008; Vazou et al. 2014]. In practice, refinement type systems often rely on SMT solvers to implement type checking. In comparison, our proposed type system is in a sweet-spot: type checking and even type inference is decidable due to existence of most general unifiers for Boolean formulas.

In summary, the main novelty of our work is the support for relational nullable types that are expressed as Boolean constraints which can be fully inferred with a novel extension of Algorithm W.

\section{CONCLUSION}

We have presented a simple, practical, and expressive relational nullable type system. The type system extends the Hindley-Milner type system with Boolean constraints, supports parametric polymorphism, and has principal types modulo Boolean equivalence. An important property of the type system is that it supports full type inference. The key insight is that the exhaustiveness condition of a relational pattern match can be translated into a Boolean formula which can be inferred because Boolean formulas have most general unifiers. Thus we can infer types with an extension of Algorithm W.

We have conducted a preliminary study on the use of relational nullability in open source projects. Three observations from the study were: (i) programmers use programming patterns where the nullability of one expression depends on the nullability of other related expressions, (ii) reasoning about such patterns requires both may and must information, and (iii) in lieu of type system support, programmers rely on run-time checks to enforce relational nullability invariants. The presented relational nullable type system regains type safety for such programming patterns while supporting full type inference.

\section{REFERENCES}

Nada Amin and Ross Tate. 2016. Java and Scala’s Type Systems are Unsound: the Existential Crisis of Null Pointers. Proc. Object-Oriented Programming, Systems, Languages, and Applications (OOPSLA) (2016).

Franz Baader. 1998. On the Complexity of Boolean Unification. Inform. Process. Lett. (1998).

Subarno Banerjee, Lazaro Clapp, and Manu Sridharan. 2019. Nullaway: Practical Type-based Null Safety for Java. In Proc. foint Symposium on European Software Engineering and the Foundations of Software Engineering (ESEC/FSE).

George Boole. 1847. The mathematical analysis of logic.

Alexandre Boudet, Jean-Pierre Jouannaud, and Manfred Schmidt-Schauß. 1989. Unification in Boolean Rings and Abelian Groups. Journal of Symbolic Computation (1989).

Dan Brotherston, Werner Dietl, and Ondřej Lhoták. 2017. Granullar: Gradual Nullable Types for Java. In Proc. International Conference on Compiler Construction (CC).

Wolfram Buttner and Helmut Simonis. 1987. Embedding Boolean Expressions into Logic Programming. Journal of Symbolic Computation (1987). 
Patrice Chalin and Perry R James. 2007. Non-Null References by Default in Java: Alleviating the Nullity Annotation Burden. In Proc. European Conference on Object-Oriented Programming (ECOOP).

Olaf Chitil. 2001. Compositional explanation of types and algorithmic debugging of type errors. In Proc. International Conference on Functional Programming (ICFP).

Luis Damas. 1984. Type Assignment in Programming Languages. Ph. D. Dissertation. The University of Edinburgh.

Manuel Fähndrich and K Rustan M Leino. 2003. Declaring and Checking Non-Null Types in an Object-Oriented Language. In Proc. Object-Oriented Programming, Systems, Languages, and Applications (OOPSLA).

Manuel Fähndrich and Songtao Xia. 2007. Establishing Object Invariants with Delayed Types. In Proc. Object-Oriented Programming, Systems, Languages, and Applications (OOPSLA).

Roger Hindley. 1969. The Principal Type-scheme of an Object in Combinatory Logic. Transactions of the American Mathematical Society (AMS) (1969).

Laurent Hubert, Thomas Jensen, and David Pichardie. 2008. Semantic Foundations and Inference of Non-Null Annotations. In International Conference on Formal Methods for Open Object-Based Distributed Systems (FMOODS).

Mark P Jones. 2003. Qualified Types: Theory and Practice. Cambridge University Press.

Leopold Löwenheim. 1908. Über das Auflösungsproblem im logischen Klassenkalkul.

Magnus Madsen and Ondřej Lhoták. 2018. Safe and Sound Program Analysis with Flix. In Proc. International Symposium on Software Testing and Analysis (ISSTA).

Magnus Madsen and Ondřej Lhoták. 2020. Fixpoints for the Masses: Programming with first-class Datalog Constraints. Proc. of the ACM on Programming Languages 4, OOPSLA (2020).

Magnus Madsen and Jaco van de Pol. 2020. Polymorphic Types and Effects with Boolean Unification. Proc. of the ACM on Programming Languages 4, OOPSLA (2020).

Magnus Madsen, Ming-Ho Yee, and Ondrej Lhoták. 2016. From Datalog to Flix: A Declarative Language for Fixed Points on Lattices. In Proc. Programming Language Design and Implementation (PLDI).

Chris Male, David J Pearce, Alex Potanin, and Constantine Dymnikov. 2008. Java Bytecode Verification for NonNull Types. In Proc. International Conference on Compiler Construction (CC).

Alberto Martelli and Ugo Montanari. 1982. An efficient unification algorithm. ACM Transactions on Programming Languages and Systems (TOPLAS) (1982).

Urusula Martin and Tobias Nipkow. 1989. Boolean Unification - The Story So Far. Journal of Symbolic Computation (1989).

Robin Milner. 1978. A Theory of Type Polymorphism in Programming. F. Comput. System Sci. (1978).

Abel Nieto, Marianna Rapoport, Gregor Richards, and Ondřej Lhoták. 2020a. Blame for Null. In Proc. European Conference on Object-Oriented Programming (ECOOP 2020).

Abel Nieto, Yaoyu Zhao, Ondřej Lhoták, Angela Chang, and Justin Pu. 2020b. Scala with Explicit Nulls. In Proc. European Conference on Object-Oriented Programming (ECOOP 2020).

Benjamin C Pierce and David N Turner. 2000. Local type inference. ACM Transactions on Programming Languages and Systems (TOPLAS) (2000).

Xin Qi and Andrew C Myers. 2009. Masked Types for Sound Object Initialization. In Proc. Principles of Programming Languages (POPL).

Patrick M Rondon, Ming Kawaguci, and Ranjit Jhala. 2008. Liquid types. In Proc. Programming Language Design and Implementation (PLDI).

Sergiu Rudeanu. 1974. Boolean Functions and Equations.

Fausto Spoto. 2008. Nullness Analysis in Boolean Form. In Proc. International Conference on Software Engineering and Formal Methods (SEFM).

Alexander J Summers and Peter Müller. 2011. Freedom Before Commitment: A Lightweight Type System for Object Initialisation. In Proc. Object-Oriented Programming, Systems, Languages, and Applications (OOPSLA).

Sam Tobin-Hochstadt and Matthias Felleisen. 2010. Logical types for untyped languages. In Proc. International Conference on Functional Programming (ICFP).

Mads Tofte and Jean-Pierre Talpin. 1997. Region-based Memory Management. Information and Computation (1997).

Niki Vazou, Eric L Seidel, Ranjit Jhala, Dimitrios Vytiniotis, and Simon Peyton-Jones. 2014. Refinement types for Haskell. In Proc. International Conference on Functional Programming (ICFP).

Philip Wadler and Stephen Blott. 1989. How to make ad-hoc polymorphism less ad hoc. In Proc. Symposium on Principles of Programming Languages (POPL).

Andrew K Wright and Matthias Felleisen. 1994. A Syntactic Approach to Type Soundness. Information and Computation (1994). 